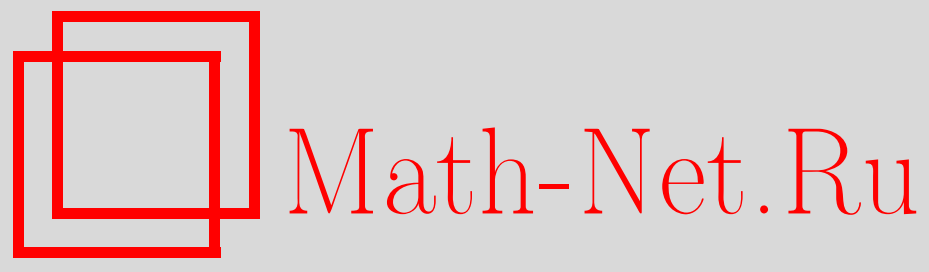

Н. Н. Вахания, В. В. Кварацхелия, Безусловная сходимость слабо субгауссовских рядов в банаховых пространствах, Теория вероятн. и ее примен., 2006, том 51, выпуск 2, 295-318

DOI: https://doi.org/10.4213/tvp55

Использование Общероссийского математического портала MathNet.Ru подразумевает, что вы прочитали и согласны с пользовательским соглашением

http: //www . mathnet.ru/rus/agreement

Параметры загрузки:

IP : 3.81 .55 .215

26 апреля 2023 г., 05:51:48

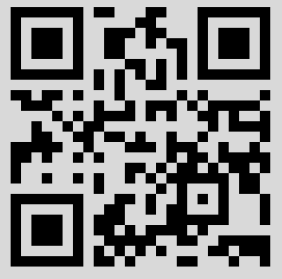




\section{БЕЗУСЛОВНАЯ СХОДИМОСТЬ СЛАБО СУБГАУССОВСКИХ РЯДОВ В БАНАХОВЫХ ПРОСТРАНСТВАХ ${ }^{1)}$}

Получены характеризации классов банаховых пространств, изоморфных $c_{0}$, а также пространств с безусловным базисом, не содержащих $l_{\infty}^{n}$ равномерно, на языке п.н. безусловной сходимости слабо субгауссовских рядов. Под п.н. безусловной сходимостью случайных рядов понимается сходимость всех перестановок данного ряда на одном и том же множестве полной вероятности. Исследуются также вопросы п.н. безусловной и слабо абсолютной сходимости в пространствах, изоморфных $c_{0}$.

Ключевые слова и фразы: банахово пространство с безусловным базисом; п.н. безусловная сходимость; субгауссовская случайная величина; слабо субгауссовский случайный элемент; строго субгауссовский случайный элемент; гауссовский случайный элемент; банахово пространство, не содержащее $l_{\infty}^{n}$ равномерно.

1. Введение. Пусть $X$ - вещественное банахово пространство, $X^{*}$ - его сопряженное. Говорят, что ряд $\sum_{k \geqslant 1} x_{k}$ сходится безусловно в пространстве $X$, если сходится ряд $\sum_{k \geqslant 1} x_{\pi(k)}$ для любой перестановки $\pi$. Мы будем пользоваться следующим критерием безусловной сходимости рядов в банаховых пространствах.

Предложение 1. Ряд $\sum_{k} x_{k}$ сходится безусловно в пространстве $X$ в том и только том случае, если выполнень следующие два условия:

(a) $\sum_{k \geqslant 1}\left|\left\langle x^{*}, x_{k}\right\rangle\right|<\infty$ для всех $x^{*} \in X^{*}$;

(b) $\lim _{n \rightarrow \infty} \sup _{\left\|x^{*}\right\| \leqslant 1} \sum_{k \geqslant n}\left|\left\langle x^{*}, x_{k}\right\rangle\right|=0$.

Известно [6], что если банахово пространство не содержит подпространства, изоморфного $c_{0}$, то выполнение условия (a) уже обеспечивает безусловную сходимость. В тех случаях, когда известно только выполнение условия (а), будем говорить, что ряд $\sum_{k \geqslant 1} x_{k}$ сходится слабо абсолютно.

* Институт вычислительной математики им. Н.И. Мусхелишвили АН Грузии, ул. Акури, 8, 0193 Тбилиси, Грузия; e-mail: vakhania@gw.acnet.ge

1) Работа выполнена при частичной поддержке грантом CRDF-GRDF № GEM1-3328-TB-03 и грантом Академии наук Грузии № 1.15.04. 
Пусть $(\Omega, \mathscr{A}, \mathbf{P})$ - вероятностное пространство, $\left(\xi_{k}\right)$ - последовательность случайных элементов со значениями в $X$ (т.е. сепарабельнозначных измеримых по Борелю отображений $\Omega$ в $X)$.

О п р е д е л е н и е. Случайный ряд $\sum_{k \geqslant 1} \xi_{k}$ сходится почти наверное (п.н.) безусловно в пространстве $X$, если найдется множество $\Omega_{0} \in \mathscr{A}$ полной вероятности $\left(\mathbf{P}\left(\Omega_{0}\right)=1\right)$ такое, что для каждого $\omega \in \Omega_{0}$ сходится любая перестановка ряда $\sum_{k \geqslant 1} \xi_{k}(\omega)$.

Заметим, что данное определение отличается от п.н. перестановочной сходимости (п.н. сходимости любой перестановки) уже в одномерном случае. Убедиться в этом можно на простом примере ряда $\sum_{k \geqslant 1} k^{-1} \gamma_{k}$, где $\left(\gamma_{k}\right)$ - последовательность независимых стандартных гауссовских случайных величин. Ясно, что этот ряд сходится п.н. перестановочно, однако не сходится п.н. безусловно, так как не сходится п.н. абсолютно.

Учитывая предложение 1 , заметим, что ряд $\sum_{k \geqslant 1} \xi_{k}$ сходится п.н. безусловно тогда и только тогда, когда найдется множество $\Omega_{0} \in \mathscr{A}$ полной вероятности такое, что для всех $\omega \in \Omega_{0}$ выполняются следующие условия: (a) $\sum_{k \geqslant 1}\left|\left\langle x^{*}, \xi_{k}(\omega)\right\rangle\right|<\infty$ для каждого $x^{*} \in X^{*}$ и (b) $\lim _{n \rightarrow \infty} \sup _{\left\|x^{*}\right\| \leqslant 1} \sum_{k \geqslant n}\left|\left\langle x^{*}, \xi_{k}(\omega)\right\rangle\right|=0$. В тех случаях, когда известно только выполнение условия (a), будем говорить, что ряд $\sum_{k \geqslant 1} \xi_{k}$ сходится п.н. слабо абсолютно.

В пространстве $c_{0}$ ряд $\sum_{k \geqslant 1} e_{k} \gamma_{k}$, где $e_{k}=(0, \ldots, 0,1,0, \ldots)$ (единица стоит на $k$-м месте), $k=1,2, \ldots$, не сходится п.н. слабо абсолютно, поскольку последовательность $\left(\gamma_{k}\right)$ не является п.н. ограниченной. Однако, как легко видеть, для всех $x^{*} \in c_{0}^{*}\left(=l_{1}\right)$ ряд $\sum_{k \geqslant 1}\left|\left\langle x^{*}, e_{k} \gamma_{k}\right\rangle\right|$ сходится на множестве полной вероятности, которое зависит от $x^{*}$.

Следуя [12], будем говорить, что случайная величина $\zeta$ является субгауссовской, если для некоторого $a, 0 \leqslant a<\infty$, и для всех вешественных чисел $t$ выполняется неравенство

$$
\mathbf{E} e^{t \zeta} \leqslant e^{a^{2} t^{2} / 2}
$$

где $\mathbf{E}$ символ математического ожидания.

Для произвольной субгауссовской случайной величины $\zeta$ через $\tau(\zeta)$ будет обозначаться число, определяемое равенством

$$
\tau(\zeta)=\inf \left\{a \in[0, \infty): \mathbf{E} e^{t \zeta} \leqslant e^{a^{2} t^{2} / 2} \text { для всех }-\infty<t<+\infty\right\} .
$$

Число $\tau(\zeta)$ называется гауссовским стандартом случайной величины $\zeta$ (в [12] используется термин гауссовское отклонение). Множество всех субгауссовских случайных величин образует банахово пространство с нормой $\tau[1]$, [2]. Легко видеть, что если $\zeta-$ субгауссовская случайная величина, то $\mathbf{E} \zeta=0$ и $\tau^{2}(\zeta) \geqslant \mathbf{E} \zeta^{2}$. Ясно, что центрированная гауссовская случайная величина $g$ с дисперсией $\sigma^{2}$ является субгауссовской и $\tau(g)=\sigma$. Отметим одно неравенство, которое устанавливает связь 
между моментами и гауссовским стандартом субгауссовской случайной величины [1]:

$$
\mathbf{E}|\zeta|^{p} \leqslant \beta_{p} \tau^{p}(\zeta), \quad p>0,
$$

где $\beta_{p}=2(p / e)^{p / 2}$. Заметим, что для значений $0<p \leqslant 2$ можно взять $\beta_{p}=1$.

Говорят, что субгауссовская случайная величина $\zeta$ является $\mathrm{cmpozo}$ субаауссовской, если $\tau(\zeta)=\left(\mathbf{E} \zeta^{2}\right)^{1 / 2}[1]$. Для произвольной случайной величины $\zeta$ обозначим

$$
\vartheta_{2}(\zeta)=\sup _{n \geqslant 1}\left(\frac{1}{n^{n}} \mathbf{E} \zeta^{2 n}\right)^{1 /(2 n)} .
$$

Можно показать [1], [2], что центрированная случайная величина $\zeta$ является субгауссовской тогда и только тогда, когда $\vartheta_{2}(\zeta)<\infty$. Кроме того, для всех субгауссовских случайных величин $\zeta$

$$
(2.05)^{-1 / 4} \sqrt{\frac{2}{e}} \tau(\zeta) \leqslant \vartheta_{2}(\zeta)^{\star} \leqslant \frac{2}{\sqrt{e}} \tau(\zeta) .
$$

Константа в левой части несколько улучшена в [3].

Случайный элемент $\xi$ в $X$ называется слабо субгауссовским, если все случайные величины $\left\langle x^{*}, \xi\right\rangle, x^{*} \in X^{*}$, являются субгауссовскими [18]. Запас слабо субгауссовских случайных элементов достаточно богат, основными распространенными примерами могут служить центрированные гауссовские и п.н. ограниченные случайные элементы.

Заметим, что определение слабой субгауссовости имеет смысл и для случая любого топологического векторного пространства $X$. Если $X$ есть векторное пространство $\mathbf{R}^{N}$ всех вещественных числовых последовательностей с тихоновской топологией, то слабая субгауссовость случайного элемента $\xi=\left(\xi_{k}\right)$ со значениями в $\mathbf{R}^{N}$ означает просто покоординатную субгауссовость в силу того, что совокупность всех субгауссовских случайных величин образует векторное пространство. Напомним, что если $X-$ (конечномерное или бесконечномерное) вещественное банахово пространство, то случайный элемент $\xi$ в $X$ называется гауссовским, если все случайные величины $\left\langle x^{*}, \xi\right\rangle, x^{*} \in X^{*}$, являются гауссовскими. Как хорошо известно, сумма двух гауссовских случайных величин не обязательно является гауссовской. Поэтому, в отличие от субгауссовского случая, последовательность числовых гауссовских случайных величин может не быть совместно гауссовской.

Слабо субгауссовский случайный элемент $\xi$ является строго субгауссовским, если $\tau^{2}\left(\left\langle x^{*}, \xi\right\rangle\right)=\mathbf{E}\left\langle x^{*}, \xi\right\rangle^{2}$ для всех $x^{*} \in X^{*}$. Очевидно, что гауссовский случайный элемент является строго субгауссовским.

Говорят, что случайный элемент $\xi$ со значениями в банаховом пространстве $X$ имеет слабый $p$-й порядок, $0<p<\infty$, если $\mathbf{E}\left|\left\langle x^{*}, \xi\right\rangle\right|^{p}<\infty$ 
для любого $x^{*} \in X^{*}$. Для случайного элемента $\xi$ со слабым первым порядком определяется математическое ожидание $\mathbf{E} \xi$ как интеграл в слабом смысле (интеграл Петтиса) следующими условиями: $\mathbf{E} \xi \in X$ и $\left\langle x^{*}, \mathbf{E} \xi\right\rangle=\mathbf{E}\left\langle x^{*}, \xi\right\rangle$ для всех $x^{*} \in X^{*}$. Для случайного элемента $\xi$ со слабым вторым порядком определяется ковариационный оператор $R_{\xi}$ следующим равенством: $\left\langle R_{\xi} x^{*}, y^{*}\right\rangle=\mathbf{E}\left\langle x^{*}, \xi-\mathbf{E} \xi\right\rangle\left\langle y^{*}, \xi-\mathbf{E} \xi\right\rangle, x^{*}, y^{*} \in X^{*}$. $R_{\xi}$ представляет собой линейный ограниченный оператор, отображающий $X^{*}$ в $X$ (обсуждение этих и связанных с ними вогросов можно найти в [4] и [17]).

Ясно, что слабо субгауссовский случайный элемент имеет как математическое ожидание, так и ковариационный оператор. Хорошо известно, что гауссовский случайный элемент со значениями в банаховом пространстве однозначно определяется своими математическим ожиданием и ковариационным оператором.

Говорят, что банахово пространство $X$ содержит $l_{\infty}^{n}$ равномерно, если для любого $\varepsilon>0$ и любого натурального $n$ найдется $n$-мерное подпространство $X_{n} \subset X$ такое, что $d\left(X_{n}, l_{\infty}^{n}\right) \leqslant 1+\varepsilon$, где $d\left(X_{n}, l_{\infty}^{n}\right)$ - так называемое расстояние Банаха-Мазура между $X_{n}$ и $l_{\infty}^{n}$. Примерами таких пространств являются пространства $c_{0}, l_{\infty}, C[0,1], \bigoplus_{l_{2}} l_{\infty}^{n}$ (заметим, что последнее из этих пространств является рефлексивным). Пространства $l_{p}, L_{p}[0,1], 1 \leqslant p<\infty$, представляют собой примеры пространств, не содержащих $l_{\infty}^{n}$ равномерно.

Пусть $X$ - банахово пространство с безусловным базисом $\left(\varphi_{i}\right)$ и $\left(\varphi_{i}^{*}\right)$ - биортогональные функционалы, соответствуюшие базису $\left(\varphi_{i}\right)$. Как известно, для всех последовательностей чисел $\left(\alpha_{i}\right)$, для которых сходится ряд $\sum_{i \geqslant 1} \alpha_{i} \varphi_{i}$, и для всех ограниченных последовательностей чисел $\left(t_{i}\right)$ имеет место оценка

$$
\left\|\sum_{i \geqslant 1} t_{i} \alpha_{i} \varphi_{i}\right\| \leqslant K \sup _{i \geqslant 1}\left|t_{i}\right|\left\|\sum_{i \geqslant 1} \alpha_{i} \varphi_{i}\right\|,
$$

где константа $K>0$ зависит только от базиса $\left(\varphi_{i}\right)$ (см. [15, с. 19]). Минимальную константу $K_{u}>0$, удовлетворяющую этому неравенству, называют константой безусловности базиса $\left(\varphi_{i}\right)$. Существует эквивалентная норма, относительно которой константа безусловности равняется единице. Поэтому при обсуждении вопросов сходимости рядов можно без ограничения общности предположить, что $K_{u}=1$.

Банахово пространство с безусловным базисом является банаховой решеткой относительно операции порядка, определяемой следуюшим образом: $\sum_{i \geqslant 1} \alpha_{i} \varphi_{i} \geqslant 0$ тогда и только тогда, когда $\alpha_{i} \geqslant 0$ для всех $i$ (см. [16, с. 2]). Модуль элемента $x=\sum_{i \geqslant 1} \alpha_{i} \varphi_{i}$ определяется следующим образом: $|x|=\sum_{i \geqslant 1}\left|\alpha_{i}\right| \varphi_{i}$ (если константа безусловности базиса $K_{u}>1$, то нужно сделать несушественную модификацию в аксиоме согласованности между порядком и нормой). Элементы $x=\sum_{i \geqslant 1} \alpha_{i} \varphi_{i}$ и 
$y=\sum_{i \geqslant 1} \beta_{i} \varphi_{i}$ пространства $X$ называются непересекающимися, если их наибольшей нижней границей является нулевой элемент. Это равносильно тому, что не пересекаются следующие подмножества натуральных чисел: $\left\{i:\left\langle\varphi_{i}^{*}, x\right\rangle \neq 0\right\}$ и $\left\{i:\left\langle\varphi_{i}^{*}, y\right\rangle \neq 0\right\}$.

В [5] было доказано, что если банахово пространство $X$ с безусловным базисом $\left(\varphi_{i}\right)$ не содержит $l_{\infty}^{n}$ равномерно, то для п.н. безусловной сходимости ряда $\sum_{k \geqslant 1} \xi_{k}$, составленного из центрированных гауссовских случайных элементов, достаточна сходимость ряда $\sum_{i \geqslant 1}\left[\sum_{k \geqslant 1}\left\langle R_{k} \varphi_{i}^{*}, \varphi_{i}^{*}\right\rangle^{1 / 2}\right] \varphi_{i}$, где $R_{k}$ - ковариационный оператор $\xi_{k}$. В настоящей статье аналогичный факт доказывается для слабо субгауссовских случайных элементов со значениями в $X$, а также устанавливается связь между п.н. безусловной сходимостью и геометрическими свойствами пространства $X$ (теоремы 1 и 2). Кроме того, приводятся некоторые достаточные условия для п.н. безусловной и слабой абсолютной сходимости слабо субгауссовских рядов в банаховых пространствах, изоморфных $c_{0}$ (теоремы 3 и 4).

\section{2. Безусловная сходимость в банаховых пространствах с} безусловным базисом, не содержащих $l_{\infty}^{n}$ равномерно. Пусть $\zeta=\left(\zeta_{k}\right)$ - последовательность субгауссовских случайных величин. На $\zeta$ можно смотреть как на случайный элемент со значениями в пространстве $\mathbf{R}^{N}$. Легко видеть, что $l_{p}, 1 \leqslant p<\infty$, является борелевским множеством в $\mathbf{R}^{N}$. Поэтому если значения $\zeta \in \mathbf{R}^{N}$ п.н. принадлежат пространству $l_{p} \subset \mathbf{R}^{N}$, то $\zeta$ можно считать случайным элементом со значениями в банаховом пространстве $l_{p}$ (см. $[17$, гл. I]). В следующей лемме даются некоторые свойства слабо субгауссовских случайных элементов со значениями в пространстве $l_{p}$.

Лемма 1. Пусть $\zeta_{k}$ - субгауссовская случайная величина с гауссовским стандартом $\tau_{k}, k=1,2, \ldots, u 1 \leqslant p<\infty$. Справедливы следующие утверждения.

(а) Последовательность случайньх величин $\zeta_{k}, k=1,2, \ldots$, является слабо субаауссовским случайньм элементом со значениями в банаховом пространстве $l_{p}$ тогда и только тогда, когда $\sum_{k \geqslant 1}\left|\zeta_{k}\right|^{p}<\infty$ n.H.

(b) Ecли $\sum_{k \geqslant 1} \tau_{k}^{p}<\infty$, mo $\sum_{k \geqslant 1}\left|\zeta_{k}\right|^{p}<\infty$ n.н.

(c) Существует слабо субгауссовский случайньй элемент $\zeta=\left(\zeta_{k}\right)$ в пространстве $l_{p}$ такой, что $\sum_{k \geqslant 1} \tau_{k}^{p}=\infty$.

(d) Ecли $\sum_{k \geqslant 1} \tau_{k}^{p}<\infty$, mo $\mathbf{E}\|\zeta\|_{l_{p}}^{t}<\infty$ dлs всех $t>0$.

(e) Ecли $\sum_{k \geqslant 1} \tau_{k}^{p}<\infty$, то для любого положительного $r$ такого, что $r^{2} \geqslant p \tau_{\zeta}^{2}$, справедливо неравенство

$$
\mathbf{P}\left\{\|\zeta\|_{l_{p}} \geqslant r\right\} \leqslant 2 e^{-r^{2} /\left(2 \tau_{\zeta}^{2}\right)}
$$

где $\tau_{\zeta}^{2}=\left(\sum_{k \geqslant 1} \tau_{k}^{p}\right)^{2 / p}$. 
(f) $E c \wedge u \sum_{k \geqslant 1} \tau_{k}^{p}<\infty, m o$

$$
\mathbf{E} e^{\varepsilon\|\zeta\|_{l_{p}}^{2}}<\infty
$$

для всех $\varepsilon<1 /\left(2 \tau_{\zeta}^{2}\right)$, где $\tau_{\zeta}^{2}=\left(\sum_{k \geqslant 1} \tau_{k}^{p}\right)^{2 / p}$.

Д оказа т ель с т в о. (а) Пусть $f=\left(f_{k}\right) \in l_{p}^{*}$ - произвольный линейный ограниченный функционал на $l_{p}\left(l_{1}^{*}=l_{\infty}\right.$ и $l_{p}^{*}=l_{q}$ при $p>1$, где $1 / p+1 / q=1)$. По неравенству Гёльдера и по условию леммы ряд $\sum_{k \geqslant 1} f_{k} \zeta_{k}$ сходится п.н. для всех $f=\left(f_{k}\right) \in l_{p}^{*}$. Кроме того, случайные величины $f_{k} \zeta_{k}$ для всех $k$ являются субгауссовскими. Как известно, пространство всех субгауссовских случайных величин является банаховым пространством [1] и, следовательно, сумма $\sum_{k \geqslant 1} f_{k} \zeta_{k}=\langle f, \zeta\rangle$ также будет субгауссовской случайной величиной.

(b) Применив теорему Б. Леви о сходимости интегралов и неравенство (1), получим соотношение

$$
\mathbf{E} \sum_{k \geqslant 1}\left|\zeta_{k}\right|^{p}=\sum_{k \geqslant 1} \mathbf{E}\left|\zeta_{k}\right|^{p} \leqslant 2\left(\frac{p}{e}\right)^{p / 2} \sum_{k \geqslant 1} \tau_{k}^{p}
$$

из которого непосредственно следует утверждение (b).

Утверждение (c) вытекает из (b) и результата в [18], согласно которому в произвольном бесконечномерном нормированном пространстве существует слабо субгауссовский случайный элемент с неинтегрируемой нормой.

(d) Достаточно показать, что $\mathbf{E}\|\zeta\|_{l_{p}}^{t}<\infty$ для всех $t \geqslant p$. Используя сначала неравенство Минковского, затем неравенство (1) и условие леммы, получим

$\mathbf{E}\|\zeta\|_{l_{p}}^{t}=\mathbf{E}\left(\sum_{k \geqslant 1}\left|\zeta_{k}\right|^{p}\right)^{t / p} \leqslant\left(\sum_{k \geqslant 1}\left(\mathbf{E}\left|\zeta_{k}\right|^{t}\right)^{p / t}\right)^{t / p} \leqslant 2\left(\frac{t}{e}\right)^{t / 2}\left(\sum_{k \geqslant 1} \tau_{k}^{p}\right)^{t / p}<\infty$.

(е) Очевидно, что $\mathbf{P}\left\{\|\zeta\|_{l_{p}} \geqslant r\right\}=\mathbf{P}\left\{\|\zeta\|_{l_{p}}^{t} \geqslant r^{t}\right\} \leqslant r^{-t} \mathbf{E}\|\zeta\|_{l_{p}}^{t}$. Полагая $t \geqslant p$ и используя оценку, полученную при доказательстве утверждения (d), получим

$$
\mathbf{P}\left\{\|\zeta\|_{l_{p}} \geqslant r\right\} \leqslant r^{-t} 2\left(\frac{t}{e}\right)^{t / 2}\left(\sum_{k \geqslant 1} \tau_{k}^{p}\right)^{t / p}=2\left(\frac{t \tau_{\zeta}^{2}}{e r^{2}}\right)^{t / 2} .
$$

Теперь выберем такое значение $t, t \geqslant p$, при котором правая часть неравенства (3) достигает своего минимального значения. Это минимальное значение есть $t_{\min }=r^{2} / \tau_{\zeta}^{2}$ и оно приводит к нужной оценке с учетом условия $t_{\min } \geqslant p$.

Утверждение (f) есть следствие утверждения (e).

Лемма 1 доказана. 
Следствие. $Е с л и \tau_{\zeta} \equiv \sum_{k \geqslant 1} \tau_{k}<\infty$, то для всех положительных $r$ справедлива следуюшая оценка вероятности хвостов распределения нормы в пространстве $l_{1}$ :

$$
\mathbf{P}\left\{\|\zeta\|_{l_{1}} \geqslant r\right\} \leqslant 2 e^{-r^{2} /\left(2 \tau_{\zeta}^{2}\right)} .
$$

Д о к а з а т е л ь с т в о. По лемме 1(е) неравенство (4) справедливо для всех $r \geqslant \tau_{\zeta}$. При $r \leqslant \tau_{\zeta}$ оно, очевидно, преврашается в тривиальное неравенство.

Теорема 1. Пусть $X$ - банахово пространство с безусловным базисом $\left(\varphi_{i}\right)$ и $\left(\varphi_{i}^{*}\right)$ - соответствующие биортогональные Функииональ. Следуюшие утверждения эквивалентнь.

(i) $X$ не содержит $l_{\infty}^{n}$ равномерно.

(ii) Из сходимости ряда

$$
\sum_{i \geqslant 1}\left[\sum_{k \geqslant 1} \tau\left(\left\langle\varphi_{i}^{*}, \xi_{k}\right\rangle\right)\right] \varphi_{i}
$$

вьтекает п.н. безусловная сходимость ряда $\sum_{k \geqslant 1} \xi_{k}$ для всех последовательностей слабо субаауссовских случайньх элементов $\left(\xi_{k}\right)$ со значениями в пространстве $X$.

(iii) Из сходимости ряда $\sum_{k \geqslant 1}\left|a_{k}\right| \tau_{k}$ вытекает сходимость ряда $\sum_{k \geqslant 1} a_{k} \zeta_{k}$ п.н. безусловно для любых числовьи субгауссовских случайных величин $\zeta_{k}$ с гауссовскими стандартами $\tau_{k}, k=1,2, \ldots, u$ всех последовательностей элементов $\left(a_{k}\right)$ в пространстве $X$.

(iv) $E c л и \zeta_{k}$ - независимые числовые субаауссовские случайнье величинь с гауссовскими стандартами $\tau_{k}, k=1,2, \ldots$, и последовательность $\left(\zeta_{k} / \tau_{k}\right)$ неограничена п.н., то из сходимости ряда $\sum_{k \geqslant 1}\left|a_{k}\right| \tau_{k}$ вытекает п.н. безусловная сходимость ряда $\sum_{k \geqslant 1} a_{k} \zeta_{k}$, где $a_{k} \in X$, $k=1,2, \ldots$.

Д ок а з а т е л с с т в о. (i) $\Rightarrow$ (ii) Так как пространство $X$ не содержит $l_{\infty}^{n}$ равномерно, оно, тем самым, не содержит подпространства, изоморфного $c_{0}$, и для доказательства данной импликации, согласно [6], достаточно найти множество $\Omega_{0} \in \mathscr{A}$ полной вероятности такое, что $\sum_{k \geqslant 1}\left|\left\langle x^{*}, \xi_{k}(\omega)\right\rangle\right|<\infty$ для всех $x^{*} \in X^{*}$ и $\omega \in \Omega_{0}$.

Предполагая сходимость ряда (5) (естественно, сходимость внутреннего ряда при каждом $i$ подразумевается) и используя лемму 1 для каждого индекса $i$, получим сходимость ряда $\sum_{k \geqslant 1}\left|\left\langle\varphi_{i}^{*}, \xi_{k}\right\rangle\right|$ на некотором множестве $\Omega_{0}^{(i)}$ полной вероятности и, следовательно, $\left(\left\langle\varphi_{i}^{*}, \xi_{k}\right\rangle\right)$, как последовательность по $k$ является случайным элементом со значениями в пространстве $l_{1}$ для каждого $i$.

Для краткости записи введем обозначения: $\left(\left\langle\varphi_{i}^{*}, \xi_{k}\right\rangle\right)=\eta_{i}$ и $\sum_{k \geqslant 1} \tau\left(\left\langle\varphi_{i}^{*}, \xi_{k}\right\rangle\right)=t_{i}, i=1,2, \ldots$. По условию теоремы ряд $\sum_{i \geqslant 1} t_{i} \varphi_{i}$ сходится в $X$ и сходимость безусловная, так как $\left(\varphi_{i}\right)$ - безусловный базис. Покажем, что в этом предположении ряд $\sum_{i \geqslant 1}\left\|\eta_{i}\right\|_{l_{1}} \varphi_{i}$ сходится п.н. 
в $X$. В самом деле, так как $X$ не содержит $l_{\infty}^{n}$ равномерно, согласно [13] сушествуют число $p \geqslant 2$, зависящее только от пространства $X$, последовательность неотрицательных чисел $\left(\alpha_{i}\right), \sum_{i \geqslant 1} \alpha_{i}^{p}<\infty$, и линейный ограниченный оператор $A: l_{p} \rightarrow X$ такие, что

$$
t_{i} \varphi_{i}=\alpha_{i} A e_{i}, \quad i=1,2, \ldots,
$$

где $\left(e_{i}\right)$ - естественный базис пространства $l_{p}$.

Мы сейчас покажем, что случайный ряд

$$
\sum_{i \geqslant 1} \alpha_{i} \frac{\left\|\eta_{i}\right\|_{l_{1}}}{t_{i}} e_{i}
$$

сходится п.н. (безусловно) в $l_{p}$ (если $t_{i}=0$, то соответствующий множитель $e_{i}$ в последнем ряде будем считать равным нулю). Для этого достаточно доказать сходимость ряда

$$
\sum_{i \geqslant 1} \alpha_{i}^{p} \frac{\mathbf{E}\left\|\eta_{i}\right\|_{l_{1}}^{p}}{t_{i}^{p}}
$$

Воспользуемся неравенством Минковского и затем неравенством (1). Получим

$$
\begin{aligned}
\mathbf{E}\left\|\eta_{i}\right\|_{l_{1}}^{p} & =\mathbf{E}\left(\sum_{k \geqslant 1}\left|\left\langle\varphi_{i}^{*}, \xi_{k}\right\rangle\right|\right)^{p} \leqslant\left(\sum_{k \geqslant 1}\left(\mathbf{E}\left|\left\langle\varphi_{i}^{*}, \xi_{k}\right\rangle\right|^{p}\right)^{1 / p}\right)^{p} \\
& \leqslant 2\left(\frac{p}{e}\right)^{p / 2}\left(\sum_{k \geqslant 1} \tau\left(\left\langle\varphi_{i}^{*}, \xi_{k}\right\rangle\right)\right)^{p} \equiv 2\left(\frac{p}{e}\right)^{p / 2} t_{i}^{p} .
\end{aligned}
$$

Следовательно,

$$
\sum_{i \geqslant 1} \alpha_{i}^{p} \frac{\mathbf{E}\left\|\eta_{i}\right\|_{l_{1}}^{p}}{t_{i}^{p}} \leqslant 2\left(\frac{p}{e}\right)^{p / 2} \sum_{i \geqslant 1} \alpha_{i}^{p}<\infty
$$

Таким образом, ряд $\sum_{i \geqslant 1} \alpha_{i}\left(\left\|\eta_{i}\right\|_{l_{1}} / t_{i}\right) e_{i}$ сходится п.н. безусловно (поскольку $\left(e_{i}\right)-$ безусловный базис) в пространстве $l_{p}$. Обозначим множество его сходимости через $\Omega_{0}^{\prime}$ и рассмотрим множество полной вероятности $\Omega_{0}=\Omega_{0}^{\prime} \bigcap_{i=1}^{\infty} \Omega_{0}^{(i)}$.

Ясно, что

$$
A\left(\sum_{i \geqslant 1} \alpha_{i} \frac{\left\|\eta_{i}\right\|_{l_{1}}}{t_{i}} e_{i}\right)=\sum_{i \geqslant 1} \alpha_{i} \frac{\left\|\eta_{i}\right\|_{l_{1}}}{t_{i}} A e_{i}=\sum_{i \geqslant 1}\left\|\eta_{i}\right\|_{l_{1}} \varphi_{i}
$$

и, следовательно, ряд $\sum_{i \geqslant 1}\left\|\eta_{i}\right\|_{l_{1}} \varphi_{i}$ будет сходиться в $X$ п.н. безусловно, как линейный ограниченный образ п.н. безусловно сходящегося ряда $\sum_{i \geqslant 1} \alpha_{i}\left(\left\|\eta_{i}\right\|_{l_{1}} / t_{i}\right) e_{i}$ 
Теперь пусть $\omega \in \Omega_{0}$ и $x^{*} \in X^{*}$. Имеем:

$$
\begin{aligned}
& \sum_{k \geqslant 1}\left|\left\langle x^{*}, \xi_{k}(\omega)\right\rangle\right| \leqslant \sum_{k \geqslant 1} \sum_{i \geqslant 1}\left|\left\langle\varphi_{i}^{*}, \xi_{k}(\omega)\right\rangle\left\|\left|\left\langle x^{*}, \varphi_{i}\right\rangle\right|=\sum_{i \geqslant 1}\right\| \eta_{i}(\omega) \|_{l_{1}}\right|\left\langle x^{*}, \varphi_{i}\right\rangle \mid \\
& \quad=\left\langle x^{*}, \sum_{i \geqslant 1} \vartheta_{i}\left(x^{*}\right)\left\|\eta_{i}(\omega)\right\|_{l_{1}} \varphi_{i}\right\rangle \leqslant\left\|\sum_{i \geqslant 1} \vartheta_{i}\left(x^{*}\right)\right\| \eta_{i}(\omega)\left\|_{l_{1}} \varphi_{i}\right\| \cdot\left\|x^{*}\right\| \\
& \quad \leqslant\left\|\sum_{i \geqslant 1}\right\| \eta_{i}(\omega)\left\|_{l_{1}} \varphi_{i}\right\| \cdot\left\|x^{*}\right\|<\infty,
\end{aligned}
$$

где было использовано обозначение $\vartheta_{i}\left(x^{*}\right)=\operatorname{sign}\left\langle x^{*}, \varphi_{i}\right\rangle, i=1,2, \ldots$, и в последнем неравенстве было учтено предположение $K_{u}=1$. На этом доказательство справедливости импликации (i) $\Rightarrow$ (ii) закончено.

(ii) $\Rightarrow$ (iii) Пусть $\xi_{k}=a_{k} \zeta_{k}$, где $a_{k} \in X$ и $\zeta_{k}$ - субгауссовская случайная величина с гауссовским стандартом $\tau_{k}$. Тогда, учитывая однородность функционала $\tau$, имеем $\tau\left(\left\langle\varphi_{i}^{*}, \xi_{k}\right\rangle\right)=\left|\left\langle\varphi_{i}^{*}, a_{k}\right\rangle\right| \tau_{k}$, и справедливость данной импликации вытекает из следующей цепочки равенств:

$$
\begin{aligned}
\sum_{i \geqslant 1}\left[\sum_{k \geqslant 1} \tau\left(\left\langle\varphi_{i}^{*}, \xi_{k}\right\rangle\right)\right] \varphi_{i} & =\sum_{i \geqslant 1}\left[\sum_{k \geqslant 1}\left|\left\langle\varphi_{i}^{*}, a_{k}\right\rangle\right| \tau_{k}\right] \varphi_{i} \\
& =\sum_{k \geqslant 1}\left[\sum_{i \geqslant 1}\left|\left\langle\varphi_{i}^{*}, a_{k}\right\rangle\right| \varphi_{i}\right] \tau_{k}=\sum_{k \geqslant 1}\left|a_{k}\right| \tau_{k},
\end{aligned}
$$

где, напомним, $\left|a_{k}\right|$ - модуль элемента $a_{k}$. Справедливость перестановки сумм легко можно обосновать критерием Коши и свойством (2) безусловных базисов.

Справедливость импликации (iii) $\Rightarrow$ (iv) очевидна.

(iv) $\Rightarrow$ (i) Напомним предположение $K_{u}=1$. Доказательство проведем от противного, т.е. предположим, что пространство $X$ содержит $l_{\infty}^{n}$ равномерно. При этом допушении построим такую последовательность $\left(a_{k}\right)$ в $X$, что ряд $\sum_{k \geqslant 1}\left|a_{k}\right| \tau_{k}$ сходится (безусловно), но ряд $\sum_{k \geqslant 1} a_{k} \zeta_{k}$ не сходится п.н. Ключевым моментом для построения такой последовательности является следующее известное утверждение (см. $[16$, с. 90]): если банахова решетка $X$ содержит $l_{\infty}^{n}$ равномерно, то для любого $\varepsilon>0$ и для любого натурального числа $r$ в решетке $X$ существует набор попарно непересекающихся элементов $x_{1}^{r}, \ldots, x_{r}^{r}$, удовлетворяющих условию

$$
\max _{1 \leqslant k \leqslant r} \beta_{k} \leqslant\left\|\sum_{k=1}^{r} \beta_{k}\left|x_{k}^{r}\right|\right\| \leqslant(1+\varepsilon) \max _{1 \leqslant k \leqslant r} \beta_{k}
$$

для всех наборов неотрицательных чисел $\beta_{1}, \ldots, \beta_{r}$.

Так как по условию $\zeta_{k}, k=1,2, \ldots$, - независимые случайные величины, по лемме Бореля-Кантелли п.н. неограниченность последовательности $\left(\zeta_{k} / \tau_{k}\right)$ равносильна выполнению условия

$$
\sum_{k \geqslant 1} \mathbf{P}\left\{\frac{\left|\zeta_{k}\right|}{\tau_{k}}>u\right\}=\infty \quad \text { для всех } \quad u>0
$$


Пусть $m_{1}$ - такое натуральное число, что

$$
\sum_{k=1}^{m_{1}} \mathbf{P}\left\{\frac{\left|\zeta_{k}\right|}{\tau_{k}}>1\right\} \geqslant 1
$$

Аналогичным образом выберем натуральные числа $m_{2}, m_{3}, \ldots$, удовлетворяющие условиям $\sum_{k=M_{i-1}+1}^{M_{i}} \mathbf{P}\left\{\left|\zeta_{k}\right| / \tau_{k}>i^{2}\right\} \geqslant 1 / i, i=2,3, \ldots$, где $M_{i}=\sum_{j=1}^{i} m_{j}, i=1,2, \ldots$

Составим разбиение $J=\left(J_{i}\right)$ множества натуральных чисел следующим образом. Пусть $\left(m_{i}\right)$ - найденная выше последовательность натуральных чисел. В первый блок $J_{1}$ поместим $m_{1}$ чисел от 1 до $m_{1}$ включительно, и, продолжая этот процесс, в $i$-й блок $J_{i}, i=2,3, \ldots$, поместим $m_{i}$ чисел от $M_{i-1}+1$ до $M_{i}$ включительно. Рассмотрим в $X$ следующую последовательность элементов $\left(b_{k}\right):$ для любого $k \in J_{i}$ (каждое натуральное число $k$ входит в один из блоков $J_{i}$ ) положим $b_{k}=x_{\bar{k}}^{m_{i}} / i^{2}$, где $\bar{k}=k-M_{i-1}$ есть порядковый номер числа $k$ в блоке $J_{i}$ и элементы $x_{1}^{m_{i}}, \ldots, x_{m_{i}}^{m_{i}}$ для каждого $m_{i}$ обладают свойством (6) с $r=m_{i}$, $i=1,2, \ldots$. Покажем, что ряд $\sum_{k \geqslant 1}\left|b_{k}\right|$ сходится. Прежде всего заметим, что в силу правого неравенства в (6) норма суммы произвольного количества элементов $b_{k}$ с индексами $k$ из любого одного блока $J_{i}$ оценивается сверху числом $(1+\varepsilon) / i^{2}$. Поэтому достаточно доказать сходимость или, что то же самое, фундаментальность подпоследовательности $S_{M_{n}}$ последовательности $\left(S_{n}\right), S_{n}=\sum_{k=1}^{n}\left|b_{k}\right|$. В силу правого неравенства в (6) имеем оценку

$$
\left\|S_{M_{n}}-S_{M_{n+p}}\right\|=\left\|\sum_{i=n}^{n+p} \frac{1}{i^{2}} \sum_{k \in J_{i}}\left|x_{\bar{k}}^{m_{i}}\right|\right\| \leqslant(1+\varepsilon) \sum_{i=n}^{n+p} \frac{1}{i^{2}},
$$

и поэтому, независимо от значений $m_{i}$, ряд $\sum_{k \geqslant 1}\left|b_{k}\right|$ сходится.

Положим теперь $a_{k}=b_{k} / \tau_{k}$. Очевидно, что ряд $\sum_{k \geqslant 1}\left|a_{k}\right| \tau_{k}$ сходится. Следовательно, по условию (iv) теоремы ряд $\sum_{k \geqslant 1} a_{k} \zeta_{k}$ должен сходиться п.н. безусловно. Но это не так, последний ряд не сходится п.н. даже в обычном смысле, поскольку по построению последовательности $\left(a_{k}\right)$ имеем:

$$
\sum_{k \geqslant 1} \mathbf{P}\left\{\left\|a_{k}\right\|\left|\zeta_{k}\right|>1\right\}=\sum_{i \geqslant 1} \sum_{k \in J_{i}} \mathbf{P}\left\{\frac{\left|\zeta_{k}\right|}{\tau_{k}}>i^{2}\right\} \geqslant \sum_{i \geqslant 1} \frac{1}{i}=\infty .
$$

Следовательно, по лемме Бореля-Кантелли последовательность $\left(a_{k} \zeta_{k}\right)$ не сходится п.н. к нулю по норме пространства $X$ и, таким образом, ряд $\sum_{k \geqslant 1} a_{k} \zeta_{k}$ не сходится даже п.н. условно. Теорема 1 доказана.

В силу того, что гауссовский случайный элемент является слабо субгауссовским случайным элементом и последовательность независимых стандартных гауссовских случайных величин является п.н. неограниченной, из доказанной теоремы вытекает следующий результат. 
Следствие. Пусть $X$ - банахово пространство с безусловнылм базисом $\left(\varphi_{i}\right),\left(\varphi_{i}^{*}\right)$ - соответствующие биортогональные функииональ, $\xi_{k}$ - чентрированные гауссовские случайные элементы со значениями в $X$ с ковариачионным оператором $R_{k}, a_{k}$ - произвольный элемент в пространстве $X,\left|a_{k}\right|-$ модуль элемента $a_{k}$ (в решетке $X)$ и $g_{k}$ - числовая стандартная гауссовская случайная величина, $k=1,2, \ldots$. Рассмотрим следующие утверждения.

(i) $X$ не содержит $l_{\infty}^{n}$ равномерно.

(ii) Ecлu

$$
\sum_{i \geqslant 1}\left[\sum_{k \geqslant 1}\left\langle R_{k} \varphi_{i}^{*}, \varphi_{i}^{*}\right\rangle^{1 / 2}\right] \varphi_{i} \quad \text { сходится в пространстве } X,
$$

то ряд $\sum_{k \geqslant 1} \xi_{k}$ сходится п.н. безусловно.

(iii) Если ряд $\sum_{k \geqslant 1}\left|a_{k}\right|$, составленный из модулей элементов $a_{k}$, сходится в пространстве $X$, то ряд $\sum_{k \geqslant 1} a_{k} g_{k}$ сходится п.н. безусловно.

Справедливы импликачии: (i) $\Rightarrow$ (ii) $\Rightarrow$ (iii).

Если рассматриваемье гауссовские случайные элементы $\xi_{k}$ независимы и независимы также случайные величины $g_{k}$, то имеет место эквивалентность (i) $\Leftrightarrow$ (ii) $\Leftrightarrow$ (iii).

Отметим, что в этом следствии не предполагается совместная гауссовость ни $\xi_{k}$, ни $g_{k}, k=1,2, \ldots$

Нетрудно убедиться в том, что если базис $\left(\varphi_{i}\right)$ не является безусловным, то выполнение условия (ii) не обеспечивает даже обычную сходимость ряда $\sum_{k \geqslant 1} \xi_{k}$ п.н. по норме пространства $X$. Соответствуюший пример построим в пространстве $l_{1}$. Рассмотрим в $l_{1}$ условный базис Шаудера $\left(\varphi_{i}\right): \varphi_{1}=e_{1}, \varphi_{i}=e_{i}-e_{i-1}, i \geqslant 2$, где $\left(e_{i}\right)$ - естественный базис пространства $l_{1}$. Легко видеть, что

$$
\sum_{k=1}^{n} \alpha_{k} \varphi_{k}=\sum_{k=1}^{n-1}\left(\alpha_{k}-\alpha_{k+1}\right) e_{k}+\alpha_{n} e_{n}, \quad n=2,3, \ldots
$$

Следовательно, ряд $\sum_{k \geqslant 1} \alpha_{k} \varphi_{k}$ сходится в $l_{1}$ тогда и только тогда, когда $\lim _{k \rightarrow \infty} \alpha_{k}=0$ и $\sum_{k \geqslant 1}\left|\alpha_{k}-\alpha_{k+1}\right|<\infty$. Теперь пусть $\xi_{k}=\alpha_{k} \varphi_{k} \gamma_{k}$, где $\alpha_{2 k-1}=(k+\dot{1}) /(k \sqrt{k}), \alpha_{2 k}=(k-1) /(k \sqrt{k}), k=1,2, \ldots$, и $\left(\gamma_{k}\right)-$ последовательность независимых стандартных гауссовских случайных величин. Условие п. (ii) теоремы 1 выполняется, так как

$$
\begin{aligned}
& \left\|\sum_{i \geqslant 1}\left[\sum_{k \geqslant 1}\left\langle R_{k} \varphi_{i}^{*}, \varphi_{i}^{*}\right\rangle^{1 / 2}\right] \varphi_{i}\right\|_{l_{1}}=\left\|\sum_{i \geqslant 1}\left|\alpha_{i}\right| \varphi_{i}\right\|_{l_{1}} \\
& =\sum_{i \geqslant 1}|| \alpha_{i}|-| \alpha_{i+1}||=\sum_{k \geqslant 1}|| \alpha_{2 k-1}|-| \alpha_{2 k}||+\sum_{k \geqslant 1}|| \alpha_{2 k}|-| \alpha_{2 k+1}|| \\
& =2 \sum_{k \geqslant 1} \frac{1}{k \sqrt{k}}+\sum_{k \geqslant 1}\left|\frac{k-1}{k \sqrt{k}}-\frac{k+2}{(k+1) \sqrt{k+1}}\right| \leqslant 3 \sum_{k \geqslant 1} \frac{1}{k \sqrt{k}}<\infty .
\end{aligned}
$$


Теперь убедимся в том, что ряд $\sum_{k \geqslant 1} \xi_{k}=\sum_{k \geqslant 1} \alpha_{k} \varphi_{k} \gamma_{k}$ не сходится п.н. Известно [8], что ряд $\sum_{k \geqslant 1} \xi_{k}$, составленный из центрированных независимых гауссовских случайных элементов со значениями в пространстве $l_{1}$, сходится п.н. тогда и только тогда, когда

$$
\sum_{i \geqslant 1}\left(\sum_{k \geqslant 1} r_{i i}(k)\right)^{1 / 2}<\infty
$$

где $\left[r_{i j}(k)\right]$ - матричное представление ковариационного оператора $R_{k}$ случайного элемента $\xi_{k}$ (т.е. $r_{i j}(k)=\left\langle R_{k} e_{i}, e_{j}\right\rangle$, где $e_{i}=(0, \ldots, 0,1,0, \ldots)$ (единица стоит на $i$-м месте) - единичные векторы в пространстве $l_{\infty}$, $i, j, k=1,2, \ldots)$. Проверим выполнение условия (7). Для этого заметим, что $r_{11}(1)=\alpha_{1}^{2}$ и

$$
r_{i i}(k)=\left\{\begin{array}{ll}
\alpha_{k}^{2} & \text { при } k=i \quad \text { и } k=i+1, \\
0 & \text { в остальных случаях, }
\end{array} \quad \text { где } i=2,3, \ldots .\right.
$$

Поэтому очевидно, что

$$
\begin{aligned}
& \sum_{i \geqslant 2}\left(\sum_{k \geqslant 1} r_{i i}(k)\right)^{1 / 2}=\sum_{i \geqslant 2}\left(\alpha_{i}^{2}+\alpha_{i+1}^{2}\right)^{1 / 2}=\sum_{j \geqslant 2}\left(\alpha_{2 j-1}^{2}+\alpha_{2 j}^{2}\right)^{1 / 2} \\
& \quad+\sum_{j \geqslant 1}\left(\alpha_{2 j}^{2}+\alpha_{2 j+1}^{2}\right)^{1 / 2} \geqslant \sum_{j \geqslant 2}\left(\frac{(j+1)^{2}}{j^{3}}+\frac{(j-1)^{2}}{j^{3}}\right)^{1 / 2} \geqslant \sum_{j \geqslant 2} \sqrt{\frac{2}{j}}=\infty
\end{aligned}
$$

и, следовательно, ряд $\sum_{k \geqslant 1} \alpha_{k} \varphi_{k} \gamma_{k}$ не сходится п.н. в пространстве $l_{1}$. Более того, легко видеть, что никакая перестановка данного ряда не сходится п.н.

\section{3. Необходимое условие безусловной сходимости в про-} странствах, изоморфных $c_{0}$. Согласно теореме 1 , в пространствах, не содержащих $l_{\infty}^{n}$ равномерно, сходимость ряда (5) является достаточным условием п.н. безусловной сходимости слабо субгауссовского ряда. Как показывает приводимый ниже пример, необходимость этого условия не имеет места даже в одномерном случае (однако для ряда, составленного из гауссовских случайных величин, сходимость ряда (5) является и необходимым условием [17]).

П р и м е р. Пусть $\zeta_{k}, k=1,2, \ldots$, - последовательность субгауссовских случайных величин с распределением

$\mathbf{P}\left\{\zeta_{k}=1\right\}=\mathbf{P}\left\{\zeta_{k}=-1\right\}=\frac{1}{2 k^{2}}, \quad \mathbf{P}\left\{\zeta_{k}=0\right\}=1-\frac{1}{k^{2}}, \quad k=1,2, \ldots$

Ясно, что ряд $\sum_{k \geqslant 1} \zeta_{k}$ сходится п.н. абсолютно. Далее, для всех натуральных $k \geqslant 2$ справедливы следующие неравенства:

$$
2 e \ln k \leqslant \inf _{n \geqslant 1}\left\{n k^{2 / n}\right\} \leqslant 2 e^{2} \ln k
$$


В самом деле, для каждого фиксированного $k$ функция $f(t)=t k^{2 / t}, t \geqslant 1$, достигает своего минимального значения, которое равно $2 e \ln k$, в точке $t=2 \ln k$. Следовательно, справедливость левого неравенства в (8) показана. Для доказательства правой части рассмотрим числа $n_{1}=[2 \ln k]$ и $n_{2}=[2 \ln k]+1([x]$ означает целую часть числа $x)$. Легко можно показать, что $\inf \{f(n), n=1,2, \ldots\}=\min \left\{f\left(n_{1}\right), f\left(n_{2}\right)\right\}$. Рассмотрим замкнутый интервал $\left[2 \ln k-\frac{1}{2}, 2 \ln k+\frac{1}{2}\right]$. Ясно, что он содержит по крайней мере одну из точек $n_{1}$ и $n_{2}$. Если $n_{1} \in\left[2 \ln k-\frac{1}{2}, 2 \ln k+\frac{1}{2}\right]$, то

$$
f\left(n_{1}\right)=n_{1} k^{2 / n_{1}} \leqslant k^{2 /\left(2 \ln k-\frac{1}{2}\right)} 2 \ln k \leqslant k^{2 / \ln k} 2 \ln k=2 e^{2} \ln k,
$$

а если $n_{2} \in\left[2 \ln k-\frac{1}{2}, 2 \ln k+\frac{1}{2}\right]$, то

$$
f\left(n_{2}\right)=n_{2} k^{2 / n_{2}} \leqslant\left(2 \ln k+\frac{1}{2}\right) k^{2 /(2 \ln k)} \leqslant 3 e \ln k<2 e^{2} \ln k,
$$

и соотношение (8) доказано.

В силу определения $\vartheta_{2}$ соотношение (8) дает $(4 e \ln k)^{-1} \leqslant \vartheta_{2}^{2}\left(\zeta_{k}\right) \leqslant$ $(2 e \ln k)^{-1}$ для каждого $k \geqslant 2$. Учитывая неравенство $(\sqrt{e} / 2) \vartheta_{2}(\zeta) \leqslant \tau(\zeta)$ для каждой субгауссовской случайной величины $\zeta$, заключаем, что $\sum_{k \geqslant 1} \tau\left(\zeta_{k}\right)=\infty$, и построение примера завершено.

Важно отметить, что в построенном примере последовательность $\left(\tau^{2}\left(\zeta_{k}\right) / \mathbf{E} \zeta_{k}^{2}\right)$ неограничена.

Основной целью настоящего пункта является доказательство того, что в пространствах, изоморфных $c_{0}$, и только в них, сходимость ряда (5) является необходимым условием п.н. безусловной сходимости для некоторого класса слабо субгауссовских рядов (в частности, гауссовских рядов). Напомним, что $c_{0}$ обозначает банахово пространство всех сходящихся к нулю вещественных числовых последовательностей с супремумнормой. Кроме того, $c_{0}$ является также банаховой решеткой относительно порядка, индуцированного естественным базисом.

Справедлив следующий результат.

Теорема 2. Пусть $X$ - банахово пространство с нормированньмм безусловным базисом $\left(\varphi_{i}\right),\left(\varphi_{i}^{*}\right)$ - соответствующие биортогональнье функииональ. Пусть, кроме того, $\left(\zeta_{k}\right)$ - последовательность числовьх субгауссовских случайньх величин с единичными дисперсиями, удовлетворяющих условию

$$
\sup _{k \geqslant 1} \tau^{2}\left(\zeta_{k}\right)<\infty
$$

Следуюшие утверждения эквивалентны.

(i) $X$ изоморфно пространству $c_{0}$.

(ii) Из п.н. безусловной сходимости ряда $\sum_{k \geqslant 1} \xi_{k}$ вылекает сходимость ряда $\sum_{i \geqslant 1}\left[\sum_{k \geqslant 1} \tau\left(\left\langle\varphi_{i}^{*}, \xi_{k}\right\rangle\right)\right] \varphi_{i}$ для всех слабо субаауссовских слу- 
чайных элементов $\xi_{k}$ со значениями в $X$ и ковариачионным оператором $R_{k}$ таких, что

$$
\tau^{2}\left(\left\langle x^{*}, \xi_{k}\right\rangle\right) \leqslant C\left\langle R_{k} x^{*}, x^{*}\right\rangle \quad \text { dлs всех } \quad x^{*} \in X^{*} \quad u \quad k=1,2, \ldots,
$$

где $C \geqslant 1$ - некоторая константа, не зависящая ни от $x^{*}$, ни от $k$.

(iii) Из п.н. безусловной сходимости ряда $\sum_{k \geqslant 1} a_{k} \zeta_{k}$ вытекает сходимость ряда $\sum_{k \geqslant 1}\left|a_{k}\right| \tau_{k}$ для всех последовательностей әлементов $\left(a_{k}\right)$ в $X$, где $\tau_{k}=\tau\left(\zeta_{k}\right), k=1,2, \ldots$.

Для доказательства этой теоремы нам потребуется ряд вспомогательных фактов.

Лемма 2. Пусть $\zeta-$ числовая случайная величина, и пусть $\partial л я$ некоторых чисел $K \geqslant 1 u p>q>0$ справедливо неравенство

$$
\left(\mathbf{E}|\zeta|^{p}\right)^{1 / p} \leqslant K\left(\mathbf{E}|\zeta|^{q}\right)^{1 / q}
$$

Тогда

$$
\left(\mathbf{E}|\zeta|^{r}\right)^{1 / r} \leqslant K^{\delta}\left(\mathbf{E}|\zeta|^{s}\right)^{1 / s}
$$

для всех $0<r, s \leqslant p$, где

$$
\delta=\left\{\begin{array}{lll}
0 & n p u \quad 0<r \leqslant s \leqslant p, \\
1 & n p u \quad q \leqslant s<r \leqslant p, \\
q(p-s)(s(p-q))^{-1} & n p u \quad 0<s<q<r \leqslant p, \\
p(q-s)(s(p-q))^{-1} & \text { npu } 0<s<r \leqslant q .
\end{array}\right.
$$

Д ок а з а т ельст в о. Учитывая монотонность $\left(\mathbf{E}|\zeta|^{t}\right)^{1 / t}$ как функции от $t, t>0$, утверждение леммы для $0<r \leqslant s \leqslant p$ и $q \leqslant s<r \leqslant p$ очевидно. Доказательство начнем с рассмотрения случая $0<s<q<r \leqslant p$. В этом случае числа $\alpha=p(q-s) /(q(p-s))$ и $\beta=s(p-q) /(q(p-s))$ удовлетворяют условиям: $0<\alpha, \beta<1$ и $\alpha+\beta=1$. Применением неравенства Гёльдера получим

$$
\mathbf{E}|\zeta|^{q}=\mathbf{E}|\zeta|^{\alpha q}|\zeta|^{\beta q} \leqslant\left(\mathbf{E}|\zeta|^{\alpha q t}\right)^{1 / t}\left(\mathbf{E}|\zeta|^{\beta q t^{*}}\right)^{1 / t^{*}}
$$

где $1<t, t^{*}<\infty$ и $1 / t+1 / t^{*}=1$. Выберем $t$ из условия $\alpha q t=p$. Ясно, что $t>1$ и

$$
t=\frac{p-s}{q-s}, \quad t^{*}=\frac{t}{t-1}=\frac{p-s}{p-q} .
$$

При таком выборе $t$ соотношение (11) дает

$$
\begin{aligned}
\mathbf{E}|\zeta|^{q} & \leqslant\left(\mathbf{E}|\zeta|^{p}\right)^{(q-s) /(p-s)}\left(\mathbf{E}|\zeta|^{s}\right)^{(p-q) /(p-s)} \\
& \leqslant K^{p(q-s) /(p-s)}\left(\mathbf{E}|\zeta|^{q}\right)^{p(q-s) /(q(p-s))}\left(\mathbf{E}|\zeta|^{s}\right)^{(p-q) /(p-s)} .
\end{aligned}
$$

Отсюда легко получить, что

$$
\left(\mathbf{E}|\zeta|^{q}\right)^{1 / q} \leqslant K^{p(q-s) /(s(p-q))}\left(\mathbf{E}|\zeta|^{s}\right)^{1 / s} .
$$


Окончательно имеем

$$
\left(\mathbf{E}|\zeta|^{r}\right)^{1 / r} \leqslant\left(\mathbf{E}|\zeta|^{p}\right)^{1 / p} \leqslant K\left(\mathbf{E}|\zeta|^{q}\right)^{1 / q} \leqslant K^{q(p-s) /(s(p-q))}\left(\mathbf{E}|\zeta|^{s}\right)^{1 / s} .
$$

Остается рассмотреть случай $0<s<r \leqslant q$, который легко сводится к предыдушему. В самом деле, применением (12) получим

$$
\left(\mathbf{E}|\zeta|^{r}\right)^{1 / r} \leqslant\left(\mathbf{E}|\zeta|^{q}\right)^{1 / q} \leqslant K^{p(q-s) /(s(p-q))}\left(\mathbf{E}|\zeta|^{s}\right)^{1 / s}
$$

что и требовалось доказать.

3 а м е ч а н и е. Лемма 2 доказана и использована в [10] для изучения свойств субгауссовских случайных векторов в банаховых пространствах. Там же отмечено, что результат леммы 2 можно извлечь из [11]. Наше доказательство этой леммы является элементарным. Кроме того, константа $K^{\delta}$, найденная в доказательстве леммы, позволяет проследить зависимость от участвующих в задаче параметров $p, q, r, s$ и $K$.

Лемма 3. Пусть $\alpha(t)$ - произвольная функиия, определенная на произвольном непустом множестве $T$, и пусть $\zeta-$ произвольная случайная величина. Тогда если $\mathbf{P}\{\zeta \geqslant \alpha(t)\} \geqslant \delta>0$ для всех $t \in T$, то

$$
\mathbf{P}\left\{\zeta \geqslant \sup _{t \in T} \alpha(t)\right\} \geqslant \delta \text {. }
$$

Д ок а з а т е ль с т в о. Пусть $\left(\varepsilon_{n}\right)$ - монотонно сходящаяся к нулю числовая последовательность и $\left(t_{n}\right)$ - последовательность элементов множества $T$ такая, что $\alpha\left(t_{n}\right)>\sup _{t \in T} \alpha(t)-\varepsilon_{n}$. По условию леммы имеем

$$
\mathbf{P}\left\{\zeta>\sup _{t \in T} \alpha(t)-\varepsilon_{n}\right\} \geqslant \mathbf{P}\left\{\zeta \geqslant \alpha\left(t_{n}\right)\right\} \geqslant \delta .
$$

Теперь, переходя к пределу при $n \rightarrow \infty$ и учитывая непрерывность вероятности, получаем доказываемое неравенство.

Лемма 4. Пусть последовательность числовых случайных величин $\left(\zeta_{k}\right)$ по вероятности стремится $\kappa$ нулю, и пусть $\alpha_{k} \geqslant 0$, $k=1,2, \ldots$ Тогда если

$$
\mathbf{P}\left\{\left|\zeta_{k}\right| \geqslant \alpha_{k}\right\} \geqslant \delta>0 \quad \text { для всех } \quad k=1,2, \ldots,
$$

$m o \lim _{k \rightarrow \infty} \alpha_{k}=0$.

Д ок а з ат ел ь с т в. Предположим противное, пусть существует число $\beta>0$ такое, что $\alpha_{k_{j}} \geqslant \beta$ для всех $j=1,2, \ldots$. Тогда имеем $\mathbf{P}\left\{\left|\zeta_{k_{j}}\right| \geqslant \beta\right\} \geqslant \mathbf{P}\left\{\left|\zeta_{k_{j}}\right| \geqslant \alpha_{k_{j}}\right\} \geqslant \delta$, что противоречит условию леммы.

Лемма 5. Пусть $X$ - банахово пространство и $\left(\zeta_{k}\right)$ - последовательность числовых случайньх величин, удовлетворяюших условию

$$
\left(\mathrm{E}\left|\zeta_{k}\right|^{2}\right)^{1 / 2} \leqslant M \mathrm{E}\left|\zeta_{k}\right| \quad \partial \Omega \text { всех } \quad k=1,2, \ldots,
$$


где константа $M \geqslant 1$ не зависит от $k$. Пусть далее $\left(a_{k}\right)-$ последовательность әлементов пространства $X$. Справедливь следующие утверждения.

(а) Из п.н. абсолютной сходимости ряда $\sum_{k \geqslant 1} \zeta_{k}$ вытекает сходимость ряда $\sum_{k \geqslant 1} \mathbf{E}\left|\zeta_{k}\right|$.

(b) Из п.н. безусловной сходимости ряда $\sum_{k \geqslant 1} a_{k} \zeta_{k}$ вытекает безусловная сходимость ряда $\sum_{k \geqslant 1} a_{k} \mathbf{E}\left|\zeta_{k}\right|$.

Д ок аз а т ельст в о. (а) Введем обозначение $\eta_{n}=\sum_{k=1}^{n}\left|\zeta_{k}\right|$, $n=1,2, \ldots$. По условию $\lim _{n \rightarrow \infty} \eta_{n}=\eta$ п.н., где $\eta=\sum_{k \geqslant 1}\left|\zeta_{k}\right|$. Пользуясь известным элементарным неравенством (см., например, $[4$, лемма 4.2 , с. 240$])$, имеем

$$
\mathbf{P}\left\{\eta_{n} \geqslant \lambda \mathbf{E} \eta_{n}\right\} \geqslant(1-\lambda)^{2} \frac{\left(\mathbf{E} \eta_{n}\right)^{2}}{\mathbf{E} \eta_{n}^{2}} \geqslant(1-\lambda)^{2} \frac{\left(\sum_{k=1}^{n} \mathbf{E}\left|\zeta_{k}\right|\right)^{2}}{\left(\sum_{k=1}^{n}\left(\mathbf{E}\left|\zeta_{k}\right|^{2}\right)^{1 / 2}\right)^{2}} \geqslant \frac{(1-\lambda)^{2}}{M^{2}}
$$

для всех $0<\lambda<1$ и $n=1,2, \ldots$ Отсюда вытекает, что последовательность $\mathbf{E} \eta_{n}=\sum_{k=1}^{n} \mathbf{E}\left|\zeta_{k}\right|, n=1,2, \ldots$, ограничена, т.е. $\sum_{k \geqslant 1} \mathbf{E}\left|\zeta_{k}\right|<\infty$.

(b) Пусть ряд $\sum_{k \geqslant 1} a_{k} \zeta_{k}$ сходится п.н. безусловно. Тогда для любого $x^{*} \in X^{*}$ п.н. сходится ряд $\sum_{k \geqslant 1}\left|\left\langle x^{*}, a_{k}\right\rangle \zeta_{k}\right|$. Очевидно, что согласно (a) сходится ряд $\sum_{k \geqslant 1}\left|\left\langle x^{*}, a_{k}\right\rangle\right| \mathbf{E}\left|\zeta_{k}\right|$. В силу предложения 1 остается показать, что

$$
\lim _{n \rightarrow \infty} \sup _{\left\|x^{*}\right\| \leqslant 1} \sum_{k \geqslant n}\left|\left\langle x^{*}, a_{k}\right\rangle\right| \mathbf{E}\left|\zeta_{k}\right|=0 .
$$

Для каждого натурального $n$ введем обозначения: $\eta_{n}\left(x^{*}\right)=$ $\sum_{k \geqslant n}\left|\left\langle x^{*}, a_{k}\right\rangle \zeta_{k}\right|$ и $\eta_{n}=\sup _{\left\|x^{*}\right\| \leqslant 1} \eta_{n}\left(x^{*}\right)$. Очевидно, что $\eta_{n}\left(x^{*}\right) \geqslant 0$. Кроме того, $\mathbf{E} \eta_{n}\left(x^{*}\right)=\sum_{k \geqslant n}\left|\left\langle x^{*}, a_{k}\right\rangle\right| \mathbf{E}\left|\zeta_{k}\right|$ и

$$
\mathbf{E} \eta_{n}^{2}\left(x^{*}\right) \leqslant\left(\sum_{k \geqslant n}\left|\left\langle x^{*}, a_{k}\right\rangle\right|\left(\mathbf{E} \zeta_{k}^{2}\right)^{1 / 2}\right)^{2} \leqslant M^{2}\left(\sum_{k \geqslant n}\left|\left\langle x^{*}, a_{k}\right\rangle\right| \mathbf{E}\left|\zeta_{k}\right|\right)^{2}
$$

для всех $x^{*} \in X^{*}$ и $n=1,2, \ldots$. Используя опять примененное выше неравенство из [4], получим

$$
\mathbf{P}\left\{\eta_{n}\left(x^{*}\right)>\lambda \mathbf{E} \eta_{n}\left(x^{*}\right)\right\} \geqslant(1-\lambda)^{2} \frac{\left(\mathbf{E} \eta_{n}\left(x^{*}\right)\right)^{2}}{\mathbf{E} \eta_{n}^{2}\left(x^{*}\right)} \geqslant \frac{(1-\lambda)^{2}}{M^{2}}>0
$$

для всех $x^{*} \in X^{*}, \lambda \in(0,1)$ и $n=1,2, \ldots$ Зафиксируем $\lambda \in(0,1)$. Тогда ясно, что

$$
\mathbf{P}\left\{\eta_{n}>\lambda \mathbf{E} \eta_{n}\left(x^{*}\right)\right\} \geqslant \frac{(1-\lambda)^{2}}{M^{2}}>0
$$

для всех $x^{*}$ из единичного шара пространства $X^{*}$ и $n=1,2, \ldots$. Согласно лемме 3 , для всех $n$ имеем

$$
\mathbf{P}\left\{\eta_{n} \geqslant \lambda \sup _{\left\|x^{*}\right\| \leqslant 1} \mathbf{E} \eta_{n}\left(x^{*}\right)\right\} \geqslant \frac{(1-\lambda)^{2}}{M^{2}}>0 .
$$


Из условия леммы вытекает, что $\lim _{n \rightarrow \infty} \eta_{n}=0$ п.н., т.е. $\eta_{n}$ стремится к нулю при $n \rightarrow \infty$ и по вероятности. Далее, в силу соотношения (13) и леммы 4 имеем

$$
\lim _{n \rightarrow \infty} \sup _{\left\|x^{*}\right\| \leqslant 1} \sum_{k \geqslant n}\left|\left\langle x^{*}, a_{k}\right\rangle\right| \mathbf{E}\left|\zeta_{k}\right|=0 .
$$

Таким образом, ряд $\sum_{k \geqslant 1} a_{k} \mathbf{E}\left|\zeta_{k}\right|$ сходится безусловно в $X$, и на этом доказательство леммы заканчивается.

Следствие 1. Пусть $X$ - банахово пространство и $\zeta_{k}-$ числовая субгауссовская случайная величина с субгауссовским стандартом $\tau_{k}$, и пусть выполняется условие $\tau_{k} \leqslant M\left(\mathbf{E} \zeta_{k}^{2}\right)^{1 / 2}$ для всех $k=1,2, \ldots$, где константа $M>0$ не зависит от $k$. Тогда если ряд $\sum_{k \geqslant 1} a_{k} \zeta_{k}$ сходится п.н. безусловно, то ряд $\sum_{k \geqslant 1} a_{k} \tau_{k}$ сходится безусловно в пространстве $X, a_{k} \in X, k=1,2, \ldots$

Д о к а з а т е л ь с т о. Согласно неравенству (1), для каждого натурального $k$ имеем $\left(\mathbf{E} \zeta_{k}^{4}\right)^{1 / 4} \leqslant 2^{5 / 4} e^{-1 / 2} \tau_{k}$. Тогда, учитывая условие доказываемого следствия, получим неравенство $\left(\mathbf{E} \zeta_{k}^{4}\right)^{1 / 4} \leqslant$ $2^{5 / 4} M e^{-1 / 2}\left(\mathbf{E} \zeta_{k}^{2}\right)^{1 / 2}$. Отсюда, применяя лемму 2 для $p=4, q=2$ и выбирая $r=2, s=1$, приходим к неравенству

$$
\left(\mathbf{E} \zeta_{k}^{2}\right)^{1 / 2} \leqslant 2^{5 / 2} M^{2} e^{-1} \mathbf{E}\left|\zeta_{k}\right|
$$

Следовательно, по лемме 5 ряд $\sum_{k \geqslant 1} a_{k} \mathbf{E}\left|\zeta_{k}\right|$ сходится безусловно, и с учетом оценки $\tau_{k} \leqslant 2^{5 / 2} M^{3} e^{-1} \mathbf{E}\left|\zeta_{k}\right|, k=1,2, \ldots$, получим безусловную сходимость ряда $\sum_{k \geqslant 1} a_{k} \tau_{k}$. Следствие 1 доказано.

Поскольку последовательность стандартных гауссовских случайных величин $\left(g_{k}\right)$ удовлетворяет условию леммы 5 , очевидна справедливость следующего утверждения.

Следствие 2 [4]. Если $\left(g_{k}\right)$ - последовательность стандартных гауссовских случайньх величин, то в произвольном банаховом пространстве $X$ из п.н. безусловной сходимости ряда $\sum_{k \geqslant 1} a_{k} g_{k}$ вытекает безусловная сходимость ряда $\sum_{k \geqslant 1} a_{k}, a_{k} \in X, k=1,2, \ldots$.

Банаховы решетки $X, Y$ являются порядково изоморфными, если они изоморфны как банаховы пространства и в качестве оператора, осуществляющего изоморфизм, можно выбрать положительный оператор с положительным обратным (оператор $T: X \rightarrow Y$ называется положительным, если $T x \geqslant 0$ для всех $x \geqslant 0, x \in X)$. Следовательно, порядково изоморфные банаховы решетки изоморфны как банаховы пространства. Обратное неверно. Дополнительные сведения по теории банаховых решеток можно найти, например, в [16].

Мы будем пользоваться рядами, построенными с помощью матриц Сильвестра. Ряды с аналогичными конструкциями ранее встречались в работах [19]-[21] и в менее явном виде также в [7], [9]. 
Матрицы Сильвестра $\mathscr{S}^{(n)}=\left[s_{k i}^{(n)}\right]$ определяются следуюшими рекуррентными соотношениями:

$$
\mathscr{S}^{(1)}=\left[\begin{array}{cc}
1 & 1 \\
1 & -1
\end{array}\right], \quad \mathscr{S}^{(n)}=\left[\begin{array}{cr}
\mathscr{S}^{(n-1)} & \mathscr{S}^{(n-1)} \\
\mathscr{S}^{(n-1)} & -\mathscr{S}^{(n-1)}
\end{array}\right], \quad n=2,3, \ldots
$$

Легко проверить, что для произвольной последовательности вещественных чисел $\alpha_{1}, \ldots, \alpha_{2^{n}}$ справедливо равенство

$$
\sum_{k=1}^{2^{n}}\left(\sum_{i=1}^{2^{n}} s_{k i}^{(n)} \alpha_{i}\right)^{2}=2^{n} \sum_{i=1}^{2^{n}} \alpha_{i}^{2}
$$

Пусть $\left(\delta_{n}\right)$ - последовательность неотрицательных чисел и $I_{n}=$ $\left\{2^{n}-1, \ldots, 2^{n+1}-2\right\}, n=1,2, \ldots$, - разбиение множества натуральных чисел. Каждое натуральное число $k$ входит в один из отрезков $I_{n}$, для $k$ из $I_{n}$ положим $\bar{k}=k-2^{n}+2$. Ясно, что $\bar{k}$ означает порядковый номер элемента $k$ в $I_{n}$. Далее, для любого натурального $n$ и для каждой матрицы Сильвестра $\mathscr{S}^{(n)}=\left[s_{k i}^{(n)}\right]$ можно построить в $X$ последовательность элементов $d_{k}$ с помощью равенств

$$
d_{k}=\delta_{n} \sum_{i \in I_{n}} s_{\frac{k}{i} \bar{i}}^{(n)} \varphi_{i}, \quad k \in I_{n}, \quad n=1,2, \ldots
$$

Ряды с такими слагаемыми будем называть рядами Сильвестра.

Лемма 6. Пусть $X$ - банахово пространство с безусловньм базисом $\left(\varphi_{i}\right),\left(d_{k}\right)$ - последовательность элементов в пространстве $X$, определенная соотношением (15), $\zeta_{k}$ - числовые субаауссовские случайнье величинь с гауссовскими стандартами $\tau_{k}, k=1,2, \ldots$ Если

$$
\sum_{n \geqslant 1} \delta_{n} 2^{n / 2}\left(\sum_{k \in I_{n}} \tau_{k}^{2}\right)^{1 / 2}\left\|\sum_{i \in I_{n}} \varphi_{i}\right\|^{1 / 2}<\infty
$$

то случайный ряд $\sum_{k \geqslant 1} d_{k} \zeta_{k}$ сходится п.н. безусловно в $X$.

Д оказатель с т о. Учитывая равенство $\sum_{k}=\sum_{n} \sum_{k \in I_{n}}$ и используя неравенство Коши во внутренней сумме, а затем соотношение (14), получим оценку

$$
\sum_{k \geqslant l}\left|\left\langle x^{*}, d_{k}\right\rangle \zeta_{k}\right| \leqslant \sum_{n \geqslant n_{l}} \delta_{n} 2^{n / 2}\left(\sum_{k \in I_{n}} \zeta_{k}^{2}\right)^{1 / 2}\left(\sum_{i \in I_{n}}\left\langle x^{*}, \varphi_{i}\right\rangle^{2}\right)^{1 / 2}
$$

где $n_{l}$ определяется из условия $2^{n_{l}}-1 \leqslant l \leqslant 2^{n_{l}+1}-2, l=1,2, \ldots$. Ввиду монотонности $\left(\mathbf{E}|\zeta|^{t}\right)^{1 / t}$ как функции от $t, t>0$, для каждого натурального $n$ справедливо соотношение

$$
\mathbf{E}\left(\sum_{k \in I_{n}} \zeta_{k}^{2}\right)^{1 / 2} \leqslant\left(\sum_{k \in I_{n}} \tau_{k}^{2}\right)^{1 / 2}
$$


Теперь нам понадобится элементарное неравенство: для любого набора векторов $x_{1}, \ldots, x_{m}$ из единичного шара нормированного пространства $X$ и для любого вектора $x^{*}$ из единичного шара сопряженного пространства $X^{*}$ при любом $p \geqslant 1$

$$
\sum_{i=1}^{m}\left|\left\langle x^{*}, x_{i}\right\rangle\right|^{p} \leqslant \max _{\vartheta_{i}= \pm 1}\left\|\sum_{i=1}^{m} \vartheta_{i} x_{i}\right\|
$$

доказательство которого следует из соотношений $\sum_{i=1}^{m}\left|\left\langle x^{*}, x_{i}\right\rangle\right|^{p} \leqslant$ $\sum_{i=1}^{m}\left|\left\langle x^{*}, x_{i}\right\rangle\right| \leqslant \max _{\vartheta_{i}= \pm 1}\left\|\sum_{i=1}^{m} \vartheta_{i} x_{i}\right\|$.

Имея в виду неравенство (18) при $p=2$ и учитывая безусловность базиса $\left(\varphi_{i}\right)$ (считая константу безусловности равной единице), легко получим следующее неравенство: для каждого натурального $l$

$$
\sup _{\left\|x^{*}\right\| \leqslant 1} \sum_{k \geqslant l}\left|\left\langle x^{*}, d_{k}\right\rangle \zeta_{k}\right| \leqslant \sum_{n \geqslant n_{l}} \delta_{n} 2^{n / 2}\left(\sum_{k \in I_{n}} \zeta_{k}^{2}\right)^{1 / 2}\left\|\sum_{i \in I_{n}} \varphi_{i}\right\|^{1 / 2} .
$$

В силу неравенства (17) и теоремы Б. Леви ряд

$$
\sum_{n \geqslant 1} \delta_{n} 2^{n / 2}\left(\sum_{k \in I_{n}} \zeta_{k}^{2}\right)^{1 / 2}\left\|\sum_{i \in I_{n}} \varphi_{i}\right\|^{1 / 2}
$$

сходится п.н. Следовательно, при $l \rightarrow \infty$ правая часть неравенства (19) сходится п.н. к нулю, и отсюда в силу предложения 1 легко следует п.н. безусловная сходимость ряда $\sum_{k \geqslant 1} d_{k} \zeta_{k}$. Лемма 6 доказана.

Д ок аз а т ель с т в о т е о ре м ы 2. (i) $\Rightarrow$ (ii) Пусть $X=c_{0}$, и пусть ряд $\sum_{k \geqslant 1} \xi_{k}$ сходится п.н. безусловно в $c_{0}$. Тогда ряд $\sum_{k \geqslant 1}\left|\xi_{k}\right|$ сходится п.н. и, следовательно,

$$
\lim _{i \rightarrow \infty} \sum_{k \geqslant 1}\left|\left\langle\varphi_{i}^{*}, \xi_{k}\right\rangle\right|=0 \quad \text { п.н., }
$$

так как базис $\left(\varphi_{i}\right)$ нормированный.

С другой стороны, для каждого $x^{*} \in X^{*}$ последовательность случайных величин $\left\langle x^{*}, \xi_{k}\right\rangle, k=1,2, \ldots$, удовлетворяет условию следствия 1 леммы 5. Следовательно, $\sum_{k \geqslant 1} \tau\left(\left\langle x^{*}, \xi_{k}\right\rangle\right)<\infty$ для всех $x^{*} \in X^{*}$. Учитывая субаддитивность $\tau\left(\left\langle x^{*}, \xi_{k}\right\rangle\right)$ как функционала от $x^{*}$ и применяя принцип равномерной ограниченности, из последнего условия имеем

$$
\sup _{\left\|x^{*}\right\| \leqslant 1} \sum_{k \geqslant 1} \tau\left(\left\langle x^{*}, \xi_{k}\right\rangle\right)<\infty .
$$

Обозначим $\eta_{i}=\sum_{k \geqslant 1}\left|\left\langle\varphi_{i}^{*}, \xi_{k}\right\rangle\right|$. Последовательность случайных величин $\eta_{i}$ является равномерно интегрируемой. В самом деле, если $p>1$, то, примененяя неравенство Минковского, неравенство (1), а затем соотношение (21), получим для каждого $i$

$$
\begin{aligned}
\mathbf{E} \eta_{i}^{p} & =\mathbf{E}\left(\sum_{k \geqslant 1}\left|\left\langle\varphi_{i}^{*}, \xi_{k}\right\rangle\right|\right)^{p} \leqslant\left(\sum_{k \geqslant 1}\left(\mathbf{E}\left|\left\langle\varphi_{i}^{*}, \xi_{k}\right\rangle\right|^{p}\right)^{1 / p}\right)^{p} \\
& \leqslant 2\left(\frac{p}{e}\right)^{p / 2}\left(\sum_{k \geqslant 1} \tau\left(\left\langle\varphi_{i}^{*}, \xi_{k}\right\rangle\right)\right)^{p} \leqslant 2\left(\frac{p}{e}\right)^{p / 2}\left(\sup _{\left\|x^{*}\right\| \leqslant 1} \sum_{k \geqslant 1} \tau\left(\left\langle x^{*}, \xi_{k}\right\rangle\right)\right)^{p}<\infty .
\end{aligned}
$$


Следовательно, можно воспользоваться теоремой Лебега о мажорируемой сходимости, согласно которой имеем с учетом (20)

$$
\lim _{i \rightarrow \infty} \mathbf{E} \eta_{i}=\lim _{i \rightarrow \infty} \mathbf{E} \sum_{k \geqslant 1}\left|\left\langle\varphi_{i}^{*}, \xi_{k}\right\rangle\right|=\mathbf{E}\left(\lim _{i \rightarrow \infty} \sum_{k \geqslant 1}\left|\left\langle\varphi_{i}^{*}, \xi_{k}\right\rangle\right|\right)=0 .
$$

Теперь доказательство импликации (i) $\Rightarrow$ (ii) нетрудно завершить, повторив рассуждения, проведенные при доказательстве следствия 1 леммы 5 , и учтя эквивалентность всех нормированных безусловных базисов в $c_{0}$.

Если $X$ изоморфно $c_{0}$, то доказательство стандартным путем сводится к рассмотренному случаю.

Справедливость импликации (ii) $\Rightarrow$ (iii) очевидна.

Доказательство (iii) $\Rightarrow$ (i) проведем от противного. Пусть пространство $X$ не изоморфно $c_{0}$, и пусть последовательность субгауссовских случайных величин $\zeta_{k}$ с гауссовским стандартом $\tau_{k}=\tau\left(\zeta_{k}\right)$ удовлетворяет условию (9) настоящей теоремы. В этих предположениях построим случайный ряд (Сильвестра) $\sum_{k \geqslant 1} d_{k} \zeta_{k}$, который будет сходиться п.н. безусловно, но ряд $\sum_{k \geqslant 1}\left|d_{k}\right| \tau_{k}$ не будет сходиться. Поскольку по допущению $X$ не изоморфно $c_{0}$, последовательность чисел $\lambda(n)=\left\|\sum_{i=1}^{n} \varphi_{i}\right\|$, $n=1,2, \ldots$, неограничена (см. $[15$, с. 120]). Следовательно, существует строго возрастающая последовательность $\left(n_{l}\right)$ натуральных чисел такая, что $\left\|\sum_{i \in I_{n}} \varphi_{i}\right\| \geqslant l^{4}$ для всех $l$. Рассмотрим теперь случайный ряд Сильвестра с элементами (15), где числовая последовательность $\left(\delta_{n}\right)$ выбрана следующим образом: $\delta_{n}=0$ для всех $n \neq n_{l}$ и

И

$$
\sum_{l \geqslant 1} \delta_{n_{l}} 2^{n_{l} / 2}\left(\sum_{k \in I_{n_{l}}} \tau_{k}^{2}\right)^{1 / 2}\left\|\sum_{i \in I_{n_{l}}} \varphi_{i}\right\|^{1 / 2}<\infty
$$

$$
\lim _{l \rightarrow \infty} \delta_{n_{l}}\left(\sum_{k \in I_{n_{l}}} \tau_{k}\right)\left\|\sum_{i \in I_{n_{l}}} \varphi_{i}\right\| \neq 0
$$

Такую последовательность $\left(\delta_{n_{l}}\right)$ можно выбрать, например, следующим образом: $\quad \delta_{n_{l}}=2^{-n_{l}}\left\|\sum_{i \in I_{n}} \varphi_{i}\right\|^{-1 / 2} l^{-2}, l=1,2, \ldots$. В силу условия (9) при таком выборе $\left(\delta_{n}\right)$ ряд $\sum_{k \geqslant 1} d_{k} \zeta_{k}$ сходится п.н. безусловно, так как выполнено условие (16) леммы 6. С другой стороны, ряд $\sum_{k \geqslant 1}\left|d_{k}\right| \tau_{k}$ расходится, поскольку в случае сходимости сумма была бы $\sum_{l \geqslant 1} \delta_{n_{l}}\left(\sum_{k \in I_{n_{l}}} \tau_{k}\right)\left(\sum_{i \in I_{n_{l}}} \varphi_{i}\right)$, что невозможно, так как общий член этого ряда не стремится к нулю в силу второго условия в (22) и условия $\mathbf{E} \zeta_{k}^{2}=1$ для всех $k$. Полученное противоречие завершает доказательство теоремы.

Заметим, что поскольку в $c_{0}$ все безусловные нормированные базисы эквивалентны, то изоморфность банаховых пространств $X$ и $c_{0}$ означает порядковую изоморфность банаховых решеток $X$ и $c_{0}$. 
Ясно, что если $\left(\xi_{k}\right)$ - последовательность центрированных гауссовских случайных элементов (совместная гауссовость не предполагается), то условие (10) теоремы 2 выполняется. Поэтому теорема 2 для гауссовских случайных элементов принимает следующий вид.

Следствие. Пусть $X$ - банахово пространство с нормированным безусловным базисом $\left(\varphi_{i}\right),\left(\varphi_{i}^{*}\right)$ - соответствуюшие биортогональнье функииональ. Пусть, кроме того, $\left(g_{k}\right)$ - последовательность стандартных гауссовских случайных величин. Следующие утверждения эквивалентны.

(i) $X$ изоморфно пространству $c_{0}$.

(ii) Из п.н. безусловной сходимости ряда $\sum_{k \geqslant 1} \xi_{k}$ в пространстве $X$ вытекает сходимость ряда $\sum_{i \geqslant 1}\left[\sum_{k \geqslant 1}\left\langle R_{k} \varphi_{i}^{*}, \varphi_{i}^{*}\right\rangle^{1 / 2}\right] \varphi_{i}$ для всех чентрированных гауссовских случайных элементов $\xi_{k}$ с ковариачионньим оператором $R_{k}, k=1,2, \ldots$.

(iii) Из п.н. безусловной сходимости ряда $\sum_{k \geqslant 1} a_{k} g_{k}$ в пространстве $X$ вытекает сходимость ряда $\sum_{k \geqslant 1}\left|a_{k}\right|$ для произвольной последовательности элементов $\left(a_{k}\right)$ в $X$.

\section{4. Достаточные условия сходимости в пространствах, изо-} морфных $c_{0}$. В заключительном пункте работы рассмотрим вопрос об условиях п.н. безусловной и слабо абсолютной сходимости слабо субгауссовских рядов в пространствах $c_{0}$ на языке гауссовских стандартов координат слагаемых.

Теорема 3. Пусть банахово пространство $X$ изоморфно $c_{0}, u$ пусть $\left(\xi_{k}\right)$ - последовательность слабо субгауссовских случайньх элементов со значениями в $X$. Тогда ряд $\sum_{k \geqslant 1} \xi_{k}$ сходится п.н. безусловно в пространстве $X$, если выполнень следующие два условия:

(a) $\tau(i) \equiv \sum_{k \geqslant 1} \tau\left(\left\langle\varphi_{i}^{*}, \xi_{k}\right\rangle\right)<\infty$ для всех $i=1,2, \ldots$,

(b) $\sum_{i \geqslant 1} \exp \left\{-\varepsilon / \tau^{2}(i)\right\}<\infty$ для всех $\varepsilon>0$,

где $\left(\varphi_{i}\right)$ - нормированный безусловный базис $X u\left(\varphi_{i}^{*}\right)$ - соответствующие биортогональнье функциональ.

Д о к а з а т ель с т в о. Сначала предположим, что $X=c_{0}$. По лемме 1 и условию (а) теоремы легко видеть, что последовательность $\eta_{i}=\left(\left\langle\varphi_{i}^{*}, \xi_{1}\right\rangle,\left\langle\varphi_{i}^{*}, \xi_{2}\right\rangle, \ldots,\left\langle\varphi_{i}^{*}, \xi_{k}\right\rangle, \ldots\right)$ для каждого $i$ является слабо субгауссовским случайным элементом со значениями в пространстве $l_{1}$. Следовательно, для каждого $i$ существует множество $\Omega_{0}^{(i)} \in \mathscr{A}$ полной вероятности, на котором

$$
\sum_{k \geqslant 1}\left|\left\langle\varphi_{i}^{*}, \xi_{k}(\omega)\right\rangle\right| \equiv\left\|\eta_{i}(\omega)\right\|_{l_{1}}<\infty, \quad \omega \in \Omega_{0}^{(i)}, \quad i=1,2, \ldots
$$

Заметим, что $\lim _{i \rightarrow \infty} \eta_{i}=0$ п.н. по норме пространства $l_{1}$. В самом деле, по лемме Бореля-Кантелли для этого достаточно показать, что 
для всех $\varepsilon>0$

$$
\sum_{i \geqslant 1} \mathbf{P}\left\{\left\|\eta_{i}\right\|_{l_{1}}>\varepsilon\right\}<\infty
$$

Согласно следствию леммы 1 , имеем

$$
\mathbf{P}\left\{\left\|\eta_{i}\right\|_{l_{1}}>\varepsilon\right\} \leqslant 2 \exp \left\{-\frac{\varepsilon^{2}}{2 \tau_{\eta_{i}}^{2}}\right\}=2 \exp \left\{-\frac{\varepsilon^{2}}{2 \tau^{2}(i)}\right\} .
$$

Теперь, учитывая условие (b) теоремы, получим сходимость ряда (23) для всех $\varepsilon>0$, и, следовательно, последовательность $\left(\eta_{i}\right)$ п.н. сходится к нулю в пространстве $l_{1}$ на некотором множестве полной вероятности $\Omega_{0}^{\prime}$.

Рассмотрим множество полной вероятности $\Omega_{0}=\Omega_{0}^{\prime} \bigcap_{i=1}^{\infty} \Omega_{0}^{(i)}$ и докажем безусловную сходимость ряда $\sum_{k \geqslant 1} \xi_{k}(\omega)$ для всех $\omega \in \Omega_{0}$. Для всех $x^{*} \in c_{0}^{*}=l_{1}$ и $\omega \in \Omega_{0}$ имеем

$$
\begin{aligned}
\sum_{k \geqslant 1}\left|\left\langle x^{*}, \xi_{k}(\omega)\right\rangle\right| & \leqslant \sum_{k \geqslant 1} \sum_{i \geqslant 1}\left|\left\langle\varphi_{i}^{*}, \xi_{k}(\omega)\right\rangle\right|\left|\left\langle x^{*}, \varphi_{i}\right\rangle\right|=\sum_{i \geqslant 1}\left\|\eta_{i}(\omega)\right\|_{l_{1}}\left|\left\langle x^{*}, \varphi_{i}\right\rangle\right| \\
& \leqslant \sup _{i \geqslant 1}\left\|\eta_{i}(\omega)\right\|_{l_{1}}\left\|x^{*}\right\|<\infty .
\end{aligned}
$$

Для каждого натурального $n$ в пространстве $l_{1}$ рассмотрим последовательность случайных элементов $\eta_{i}^{(n)}=\left(0, \ldots, 0,\left\langle\varphi_{i}^{*}, \xi_{n}\right\rangle,\left\langle\varphi_{i}^{*}, \xi_{n+1}\right\rangle, \ldots\right)$, где нули стоят на первых $(n-1)$ местах. Покажем, что

$$
\lim _{n \rightarrow \infty} \sup _{i \geqslant 1}\left\|\eta_{i}^{(n)}(\omega)\right\|_{l_{1}}=0 \quad \text { для всех } \omega \in \Omega_{0} .
$$

В самом деле, если это не так, то существуют такое $\omega_{0} \in \Omega_{0}$ и такое положительное число $\delta>0$, что для бесконечно многих индексов $n$ имеем $\sup _{i \geqslant 1}\left\|\eta_{i}^{(n)}\left(\omega_{0}\right)\right\|_{l_{1}}>\delta$, т.e. $\sup _{i \geqslant 1} \sum_{k=n}^{\infty}\left|\left\langle\varphi_{i}^{*}, \xi_{k}\left(\omega_{0}\right)\right\rangle\right|>\delta$. Отсюда вытекает, что $\sum_{k \geqslant 1}\left|\left\langle\varphi_{i}^{*}, \xi_{k}\left(\omega_{0}\right)\right\rangle\right|>\delta$ для бесконечно многих индексов $i$, что противоречит условию $\lim _{i \rightarrow \infty}\left\|\eta_{i}(\omega)\right\|_{l_{1}}=0$ для всех $\omega \in \Omega_{0}$. Следовательно, для всех $\omega \in \Omega_{0}$ имеем

$$
\begin{aligned}
& \lim _{n \rightarrow \infty} \sup _{\left\|x^{*}\right\|_{l_{1}} \leqslant 1} \sum_{k \geqslant n}\left|\left\langle x^{*}, \xi_{k}(\omega)\right\rangle\right| \leqslant \lim _{n \rightarrow \infty} \sup _{\left\|x^{*}\right\|_{l_{1}} \leqslant 1} \sum_{k \geqslant n} \sum_{i \geqslant 1}\left|\left\langle\varphi_{i}^{*}, \xi_{k}(\omega)\right\rangle\right|\left|\left\langle x^{*}, \varphi_{i}\right\rangle\right| \\
& =\lim _{n \rightarrow \infty} \sup _{\left\|x^{*}\right\|_{l_{1}} \leqslant 1} \sum_{i \geqslant 1}\left\|\eta_{i}^{(n)}(\omega)\right\|_{l_{1}}\left|\left\langle x^{*}, \varphi_{i}\right\rangle\right| \leqslant \lim _{n \rightarrow \infty} \sup _{i \geqslant 1}\left\|\eta_{i}^{(n)}(\omega)\right\|_{l_{1}}=0 . \quad \text { (25) }
\end{aligned}
$$

Соотношения (24) и (25), согласно предложению 1 , показывают п.н. безусловную сходимость ряда $\sum_{k \geqslant 1} \xi_{k}$ в пространстве $c_{0}$.

Если $X$ изоморфно $c_{0}$, то доказательство стандартным путем сводится к уже рассмотренному случаю $X=c_{0}$. Теорема 3 доказана.

Легко видеть, что условия теоремы 3 не являются необходимыми. Соответствующим примером является ряд $\sum_{k \geqslant 1} \xi_{k}$ в пространстве $c_{0}$, где $\xi_{k}=\alpha_{k} e_{k} g,\left(e_{k}\right)$ - естественный базис в $c_{0}, g$ - стандартная 
гауссовская случайная величина и последовательность $\left(\alpha_{k}\right)$ выбрана таким образом, что $\lim _{k \rightarrow \infty} \alpha_{k}=0$, но не выполняются условия теоремы (в качестве такой последовательности можно взять, например, $\left.\alpha_{k}=1 / \sqrt{\ln (k+1)}\right)$.

Как известно [6], в пространствах, не содержащих подпространства, изоморфного $c_{0}$ (в частности, в пространствах, не содержащих $l_{\infty}^{n}$ равномерно), и только в них, ряд сходится безусловно тогда и только тогда, когда он сходится слабо абсолютно. В частности, в пространстве $c_{0}$ ряд, составленный из его естественного базиса, очевидно, сходится слабо абсолютно, но не сходится никакая его перестановка. Следующая теорема дает достаточное условие п.н. слабой абсолютной сходимости слабо субгауссовского ряда в пространствах, изоморфных $c_{0}$.

Теорема 4. Пусть банахово пространство $X$ изоморфно $c_{0}, u$ пусть $\left(\xi_{k}\right)$ - последовательность слабо субаауссовских случайньх элементов со значениями в $X$. Тогда ряд $\sum_{k \geqslant 1} \xi_{k}$ сходится п.н. слабо абсолютно в пространстве $X$, если выполнень следующие два условия:

(a) $\tau(i) \equiv \sum_{k \geqslant 1} \tau\left(\left\langle\varphi_{i}^{*}, \xi_{k}\right\rangle\right)<\infty$ для всех $i=1,2, \ldots$,

(b) $\sum_{i \geqslant 1} \exp \left\{-\delta / \tau^{2}(i)\right\}<\infty$ для некоторого $\delta>0$,

где $\left(\varphi_{i}\right)$ - нормированный безусловный базис $X u\left(\varphi_{i}^{*}\right)-$ coответствуюшие биортогональные Функциональ.

Д о к а з а т е л ь с т в о совпадает с доказательством предыдущей теоремы, разница только в том, что для п.н. слабой абсолютной сходимости ряда $\sum_{k \geqslant 1} \xi_{k}$ достаточно показать п.н. ограниченность последовательности случайных элементов $\left(\eta_{i}\right)$. Это также можно показать с помощью леммы Бореля-Кантелли. Сходимость для некоторого $\delta>0$ ряда $\sum_{k \geqslant 1} \mathbf{P}\left\{\left\|\eta_{i}\right\|_{l_{1}}>\delta\right\}$ проверяется так же, как в предыдушей теореме. Слабая п.н. абсолютная сходимость ряда $\sum_{k \geqslant 1} \xi_{k}$ является следствием п.н. ограниченности последовательности $\left(\eta_{i}\right)$ в силу рассуждений, примененных при получении соотношения (24).

Условия теоремы 4 не являются необходимыми. Соответствующим примером является ряд $\sum_{k \geqslant 1} \xi_{k}$ в пространстве $c_{0}$, где $\xi_{k}=e_{k} g$.

Приведем примеры п.н. безусловно и слабо абсолютно сходящихся нетривиальных случайных рядов в пространстве $c_{0}$.

Пусть $\left(g_{k}\right)$ - последовательность стандартных гауссовских случайных величин, $\left(\alpha_{k}\right)$ - последовательность вещественных чисел. По теореме 3 при $\alpha_{k}=1 / \ln (k+1)$ случайный ряд

$$
\sum_{k \geqslant 1} \alpha_{k} e_{k} g_{k}
$$

сходится п.н. безусловно в $c_{0}$ и, очевидно, не сходится п.н. абсолютно. А при $\alpha_{k}=1 / \sqrt{\ln (k+1)}$ ряд $(26)$, согласно теореме 4 , сходится п.н. слабо абсолютно, в то время как при дополнительном предположении независимости $\left(g_{k}\right)$ не сходится п.н. никакая его перестановка (см. $[17$, с. 48]). 


\section{СПИСОК ЛИТЕРАТУРЫ}

1. Булдыгин В.В., Козаченко Ю.В. О субгауссовских случайных величинах. Укр. матем. журн., 1980, т. 32, № 6, с. 723-730.

2. Булдыгин В. В., Козаченко Ю. В. Метрические характеристики случайных величин и процессов. Киев: ТВіМС, 1998, 290 с.

3. Вахания Н. Н., Квараихелия В. В., Тариеладзе В.И. Слабо субгауссовские случайные элементы в банаховых пространствах. - Укр. матем. журн., 2005, т. 57, № 9, с. 1187-1208.

4. Вахания Н. Н., Тариеладзе В. И., Чобанян С. А. Вероятностные распределения в банаховых пространствах. М.: Наука, 1985, 368 с.

5. Кварацхелия В. В. Безусловная сходимость гауссовских случайных рядов в банаховых пространствах. - Теория вероятн. и ее примен., 2000, т. 45, в. 1, с. 178-182.

6. Bessaga C., Petczyński A. On bases and unconditional convergence of series in Banach spaces. - Studia Math., 1958, v. 17, № 2, p. 151-164.

7. Bagby R., Swartz Ch. Projective tensor product of $l^{p}$-valued measures. - Mat. Casopis Sloven. Akad. Vied, 1975, v. 25, № 3, p. 265-269.

8. Chobanjan S.A., Tarieladze V.I. Gaussian characterizations of certain Banach spaces. - J. Multivariate Anal., 1977, v. 7, № 1, p. 183-203.

9. Dudley R. M., Pakula L. A counter-example on the inner product of measures. Indiana Univ. Math. J., 1971/72, v. 21, № 9, p. 843-845.

10. Fukuda $R$. Exponential integrability of sub-Gaussian vectors. - Probab. Theory Related Fields, 1990, v. 85, № 4, p. 505-521.

11. Kadec M.I., Pelczynski A. Bases, lacunary sequences and complemented subspaces in the spaces $L_{p}$. - Studia Math., 1961/62, v. 21, p. 161-176.

12. Kahane J.P. Propriétés locales des fonctions à séries de Fourier aléatories. - Studia Math., 1960, v. 19, № 1, p. 1-25.

13. Kvaratskhelia $V . V$. On unconditional convergence of random series in Banach spaces. - Lecture Notes in Math., 1980, v. 828, p. 162-166.

14. Kvaratskhelia $V . V$. Unconditional convergence of random series and the geometry of Banach spaces. - Georgian Math. J., 2000, v. 7, № 1, p. 85-96.

15. Lindenstrauss J., Tzafriri L. Classical Banach Spaces. I. Sequence Spaces. Berlin: Springer-Verlag, 1977, $188 \mathrm{p}$.

16. Lindenstrauss J., Tzafriri L. Classical Banach Spaces. II. Function Spaces. Berlin: Springer-Verlag, 1979, 243 p.

17. Vakhania N. N. Probability Distributions on Linear Spaces. New York-Amsterdam: North-Holland, 1981, 123 p.

18. Vakhania $N$. On subgaussian random vectors in normed spaces. - Bull. Georgian Acad. Sci., 2001, v. 163, № 1, p. 8-10.

19. Vakhania $N$., Kvaratskhelia V. Absolute and unconditional convergence in $l_{1}$. - Bull. Georgian Acad. Sci., 1999, v. 160, № 2, p. 201-203.

20. Vakhania N., Kvaratskhelia $V$. On a criterion for unconditional convergence of Hadamard series in the spaces $l_{p}, 1 \leqslant p<\infty$. - Bull. Georgian Acad. Sci., 2000, v. 162, № 2, p. 199-202.

21. Vakhania N., Kvaratskhelia V. Convergence of Sylvester series in Banach spaces $l_{p}$, $1 \leqslant p<\infty$. - Bull. Georgian Acad. Sci., 2001, v. 164, № 1, p. 7-9. 